\title{
Appearance of Temporal and Spatial Chaos in an Ecological System: A Mathematical Modeling Study
}

\author{
S. N. Raw ${ }^{1}$ (1) P. Mishra ${ }^{2}$ - B. P. Sarangi ${ }^{1}$ B. Tiwari ${ }^{1}$
}

Received: 27 January 2021 / Accepted: 20 April 2021 / Published online: 7 May 2021

(C) Shiraz University 2021

\begin{abstract}
The ecological theory of species interactions rests largely on the competition, interference, and predator-prey models. In this paper, we propose and investigate a three-species predator-prey model to inspect the mutual interference between predators. We analyze boundedness and Kolmogorov conditions for the non-spatial model. The dynamical behavior of the system is analyzed by stability and Hopf bifurcation analysis. The Turing instability criteria for the Spatio-temporal system is estimated. In the numerical simulation, phase portrait with time evolution diagrams shows periodic and chaotic oscillations. Bifurcation diagrams show the very rich and complex dynamical behavior of the non-spatial model. We calculate the Lyapunov exponent to justify the dynamics of the non-spatial model. A variety of patterns like interference, spot, and stripe are observed with special emphasis on Beddington-DeAngelis function response. These complex patterns explore the beauty of the spatio-temporal model and it can be easily related to real-world biological systems.
\end{abstract}

Keywords Chaos $\cdot$ Turing instability $\cdot$ Patter formulation $\cdot$ Mutual interference

\section{Introduction}

Ecological systems can be delineated by the interactions of different biological populations within a vacillating natural environment. When a similar kind of species lives in a particular geographical area, then they are identified as populations. Population is related to each other by the food they eat and distributed in different trophic levels of the food chain. Predator-prey interaction is a crucial component of the food chain system. The study of mathematical models is very informative in understanding the dynamic

\section{S. N. Raw}

sharaw.maths@nitrr.ac.in

P. Mishra

purunitrr@gmail.com

B. P. Sarangi

bpsarangi04@gmail.com

B. Tiwari

barkhatiwari72@gmail.com

1 Department of Mathematics, National Institute of Technology Raipur, Raipur, Chhattisgarh 492010, India

2 Institute of Applied Mathematics and Mechanics, University of Warsaw, ul. Banacha 2, 02-097 Warsaw, Poland behavior of predator-prey interaction. Predator-prey models have become a very extensive field of research in mathematical ecology following the pioneering work of Lotka (1924) and Volterra (1926). Interaction between predator and its prey is very crucial factor in predator-prey models and it is well known as functional response in mathematical ecology. Holling (1965) introduced four different functional responses known as Holling I, II, III \& IV type functional response.

The addition of mutual interference between species devises the foremost distinction between the BeddingtonDeAngelis (Beddington 1975; DeAngelis et al. 1975) and Holling type II functional response. Sometimes in search of food and habitat, species undergo self-inhibition along with corporation among themselves. Also, It is regarded that at high predator density, the feeding rate is relatively low due to mutual interference in predators and the prey dependent functional form ineffective to explain this. In that situation, the Beddington-DeAngelis function describes the real aspect of the ecological systems. Also, this functional response regards handling and interference as exclusive ventures by species. The only drawback of this functional response is that it explicates singular behaviour at the low population density. 
Here, both Holling type II and Beddington-DeAngelis functional responses are incorporated for modeling the interaction among populations in this paper. Naji and Balasim (2007) investigated the chaotic dynamics of a prey-predator model using the Beddington-DeAngelis type functional response. The influence of predator interference on the stability of a food chain model is studied by Upadhyay et al. (2013). A lot of work has been done to observe the dynamics induced by interference between species in predator-prey systems. Dimitrov and Kojouharov (2005) analyzed a two species prey-predator model with Beddington-DeAngelis functional response and stated that mutual interference of predator has a stabilizing effect in presence of linear prey growth. Hwang (2003) proved the global stability of a Beddington-DeAngelis type preypredator model using the divergence criterion whereas Chen et al. (2008) used Fluctuation Lemma to prove the same. Haiyin and Yasuhiro (2011) inscribed a detail comparative study of the density-dependent predator-prey model with time delay and without delay in presence of Beddington-DeAngelis functional response. Cui and Takeuchi (2006) mainly focused on species extinction criteria and permanence of a prey-predator system. Zhang et al. (2005) studied the dynamical behavior of a Holling I prey-predator model with mutual interference in the case of pest-free periodic solutions. Parshad et al. (2016) stated that mutual interference between species can cause population explosion and mathematically termed this as finite time blow up.

Mathematical modeling is adapted to demonstrate reallife predicaments and to exert prospective enhancements accordingly. Researchers practice numerous techniques to determine the numerical and analytical solution to these mathematical models. This physical interpretation of reallife problems contributes considerably to society. Epidemiological models have been used to describe HIV infection, cancer, and recently the spread of corona virus. Ali et al. (2020) studied HIV-1 infection of $C D 4^{+}$T-cells using fractional mathematical model. Sánchez et al. (2020) illustrated the mathematical pattern of the nervous stomach model and this model is designed using the differential transformation (DT) method. Gao et al. (2020a, b, c) studied the epidemic outbreaks of the noble corona virus. Yel et al. (2020) and Gao et al. (2020d) investigated the popular shallow water equations. Baskonus (2016, 2019) examined acoustic wave behaviors of Davey-Stewartson equation using the sine-Gordon expansion method, further elucidated the Boussinesq dynamical model applying the modified exponential function method.

In the nineteenth century, most mathematical models in population ecology arrogated with the uniform population distribution of species in their habitat (Legendre and Fortin
1989). The consequences of their interaction with inflammable dynamics are the subject analysis with reactiondiffusion models. Here the reaction is enthralled to predator-prey class, and diffusion leads to the redistribution of their populations. Hence species movement and interaction often give rise to the formation of a spatial pattern in their homogeneous environment. The concept of Turing instability was introduced by Turing (1852) to dispense the interaction of species in a homogeneous environment. Levin and Jackson (1972) first employed the concept of Turing pattern formation in the spatiotemporal system and later, extended by Levin and Segel (1976).

In the past quarter-century, many researchers (Alonso et al. 2002; Banerjee and Abbas 2015; Banerjee and Volpert 2017; Banerjee and Banerjee 2012; Sabrina and Araujo 2007) studied two species model systems for pattern formulation in one and two-dimensional space. Gauss (1935) studied stabilization scenario and the long-term existence of certain species in the spatial domain. $\mathrm{He}$ observed that diffusivity of the population has the power to stabilize as well as destabilize the coexistence states. Extension properties, persistence and effect of spatial distribution on the stability of population was studied by Luckinbill (1973). Liu et al. (2008) studied a spatiotemporal phytoplankton-zooplankton model with additive noise and periodic forcing. Gazi (2012) studied the role of diffusive instability of a marine plankton system. Nowadays a wide variety of literature is available evidencing spatial patterns like the spiral, spot, target and spatiotemporal chaos (Cantrell and Cosner 2003; Malchow 1993; Pascual 1993). Upadhyay et al. (2009) observed a wave of chaos (WOC) in the plankton fish model and confirm the generation of two-dimensional time-independent spatial patterns due to the wave of chaos.

Recently, some researchers (Parshad et al. 2014; Wonlyul and Inkyung 2013; Zhifu 2012) studied Turing instability and the formation of patterns for two and more interacting species. Maionchi et al. (2006) studied the complex dynamics in the spatiotemporal three species food chain model due to the diffusion of species. They observed very complex patterns in the food chain model. Baghel and Dhar (2014) performed a high-order stability analysis in a three-species food chain model. Guin et al. (2017) derived essential condition for Hopf and Turing bifurcations in a predator-prey model. Also, the study complied with self and cross-diffusion patterns. Upadhyay et al. (2013) studied dynamics induced by the predator interference with generalist predator and Parsad et al. (2016) extended this model and pointed out some other properties of predator interference. They also showed the spatial chaos and Turing instability for the model. Enlightened by the above literature, we have proposed and analyzed a food chain model including a specialist top predator. We have 
attempted to study the effect of mutual interference between two predators over the prey population. A very limited number of articles have published focusing on pattern formulation in three species predator-prey system. Therefore, in this study, the spatial complexity of the model is also investigated with the help of pattern formulation.

The paper is ascertained as follows: Sect. 2, carries the description of the proposed predator-prey model system. Conditions for boundedness, Kolmogorov and stability of model (3) is presented in Sect. 3. Section 4 deals with stability and Turing instability conditions of spatial model system (2). Numerical results are intricately exhibited in Sect. 5 with the help of MATLAB. Section 6 comprises the summed results of our model.

\section{Description of Model System}

Here we have considered a food chain model with prey and two predators. The prey is assumed as the primary consumer whereas predators are assumed to be secondary and tertiary consumers, respectively. The secondary consumer feed on prey $U$ according to the Holling type II functional response and tertiary consumer $W$ feeds on $V$ according to the Beddington-DeAngelis type functional response. Prey $(U)$ has a logistic growth delineated by the functional expression $a_{1} U\left(1-\frac{U}{K}\right)$, including intrinsic growth parameter $a_{1}$ and carrying capacity $K$. Secondary consumers $(V)$ has a decline in population density with a death rate of $a_{2}$ and $c$ expresses the death rate of tertiary consumer $(W)$. Usually, it is seen that species diffuse or show movement in the exploration of food, mate, habitat, and sometimes in fear of predators. Thus, to explain the movement of species $(U, V, W)$ in the $x-y$ direction, we have induced diffusion to the model system.

Now the assumed three species reaction-diffusion food chain model is represented as:

$$
\left\{\begin{array}{c}
\frac{\partial U}{\partial T}=a_{1} U\left(1-\frac{U}{K}\right)-\frac{e U V}{(U+D)}+D_{u} \nabla^{2} U \\
\frac{\partial V}{\partial T}=-a_{2} V+\frac{w_{1} U V}{\left(U+D_{1}\right)}-\frac{w_{2} V W}{\left(V+D_{2}+b W\right)} \\
\quad+D_{v} \nabla^{2} V \\
\frac{\partial W}{\partial T}=-c W+\frac{w_{3} V W}{\left(V+D_{2}+b W\right)}+D_{w} \nabla^{2} W
\end{array}\right.
$$

with initial conditions

$U(x, y, 0)>0, V(x, y, 0))>0, W(x, y, 0)>0$, for $(x, y) \in \Omega$, and boundary conditions $\frac{\partial U}{\partial n}=\frac{\partial V}{\partial n}=\frac{\partial W}{\partial n}=0$ for $(x, y) \in \partial \Omega, t>0$.

In model (1), $\frac{V}{\left(V+D_{2}+b W\right)}$ represents the Beddington-DeAngelis functional response with predator interference parameter $(b) \cdot \frac{U}{(U+D)}$ and $\frac{U}{\left(U+D_{1}\right)}$ express the Holling type II functional response. $D$ and $D_{1}$ symbolizes the assistance of the environment given to prey species where $D$ reveals the advantage of prey to evade predation and $D_{1}$ describes the loss in maximum gain of secondary consumer $Y$. The authenticity of this kind of situation in the environment is also given by Upadhyay et al. (2013). Ecological importance and units of adapted positive parameter values are expressed in the Table 1 . We have assumed $D_{u}, D_{v}$ and $D_{w}$ as the diffusion coefficients as the associated species actively diffuses in the $x-y$ direction. $n$ shows the outward normal to $\partial \Omega$. Here $\nabla^{2}=\frac{\partial^{2}}{\partial x^{2}}+\frac{\partial^{2}}{\partial y^{2}}$ is the Laplacian operator in $2 D$ Cartesian coordinate system $(x, y)$.

The non-dimensional model of system (1) is represented as:

$$
\left\{\begin{aligned}
\frac{\partial u}{\partial t}= & u(1-u)-\frac{u v}{\left(u+w_{4}\right)}+d_{1} \nabla^{2} u \\
\frac{\partial v}{\partial t}= & -w_{5} v+\frac{w_{6} u v}{\left(u+w_{7}\right)}-\frac{v w}{\left(v+w_{8} w+w_{9}\right)} \\
& +d_{2} \nabla^{2} v \\
\frac{\partial w}{\partial t}= & -w_{10} w+\frac{w_{11} v w}{\left(v+w_{8} w+w_{9}\right)}+d_{3} \nabla^{2} w
\end{aligned}\right.
$$

where $t=a_{1} T, u=\frac{U}{K}, v=\frac{e V}{a_{1} K}, w=\frac{e w_{2} W}{a_{1}^{2} K}, w_{5}=\frac{a_{2}}{a_{1}}, w_{4}=\frac{D}{K}$, $w_{7}=\frac{D_{1}}{K}, w_{9}=\frac{W D_{2}}{a_{1} K}, w_{6}=\frac{w_{1}}{a_{1}}, w_{11}=\frac{w_{3}}{a_{1}}, w_{8}=\frac{a_{1} b}{w_{2}}, w_{10}=\frac{c}{a_{1}}$, $d_{1}=\frac{D_{u}}{a_{1} L^{2}}, d_{2}=\frac{D_{v}}{a_{1} L^{2}}, d_{3}=\frac{D_{w}}{a_{1} L^{2}}$. Corresponding to system (2), the initial populations and no-flux boundary conditions transform to

$$
\begin{aligned}
& u(x, y, 0)>0, v(x, y, 0)>0, w(x, y, 0)>0, \\
& \text { for }(x, y) \in \Omega, \\
& \frac{\partial u}{\partial n}=\frac{\partial v}{\partial n}=\frac{\partial w}{\partial n}=0 \text { for }(x, y) \in \partial \Omega, t>0 .
\end{aligned}
$$

In the next section, we will analytically examine the behavior of the non-spatial system (3).

\section{Analysis of Temporal System}

Here, we study the model (2) without diffusion and the transformed food chain model is given by the following system of autonomous ordinary differential equation: 
Table 1 Description of Model (1) Parameters

\begin{tabular}{lll}
\hline Var/para & Units & Description \\
\hline$T$ & Time & Time \\
$U$ & Biomass & Population density of prey \\
$V$ & Biomass & Population density of secondary consumer \\
$W$ & Biomass & Population density of tertiary consumer \\
$a_{1}$ & Per day & Intrinsic growth rate of prey \\
$a_{2}$ & Per day & Death rate of $V$ \\
$K$ & Biomass & Carrying capacity of environment in support of prey \\
$e$ & Per day & Reduction rate of $U$ due to predation \\
$w_{1}$ & Per day & Maximum gain of $V$ due to consumption of prey $U$ \\
$D$ & Biomass & Half saturation constant of prey $U$ at which reduction rate is half of $e$ \\
$D_{1}$ & Biomass & Half saturation constant of prey $U$ at which gain in $V$ is half of $w_{1}$ \\
$w_{2}$ & Per day & Reduction rate of $V$ due to predation \\
$w_{3}$ & Per day & Maximum gain of $W$ due to consumption of $V$ \\
$D_{2}$ & Biomass & Normalizing coefficient to the environment for interaction of $V$ and $W$ \\
$b$ & Per day & Predator interference parameter \\
$c$ & Per day & Death rate of $W$ \\
\hline
\end{tabular}

$$
\left\{\begin{aligned}
\frac{\mathrm{d} u}{\mathrm{~d} t} & =u(1-u)-\frac{u v}{\left(u+w_{4}\right)}=u g_{1}(u, v, w), \\
\frac{\mathrm{d} v}{\mathrm{~d} t}= & -w_{5} v+\frac{w_{6} u v}{\left(u+w_{7}\right)}-\frac{v w}{\left(v+w_{8} w+w_{9}\right)} \\
& =v g_{2}(u, v, w) \\
\frac{\mathrm{d} w}{\mathrm{~d} t}= & -w_{10} w+\frac{w_{11} v w}{\left(v+w_{8} w+w_{9}\right)}=w g_{3}(u, v, w) .
\end{aligned}\right.
$$

We have limited the non-dimensionalized system (3) with only eight parameters. Here $u g_{1}, v g_{2}$ and $w g_{3}$ are continuous smooth functions and Lipschizian on $R_{+}^{3}$ $=\{(u, v, w): u(0)>0, v(0)>0, w(0)>0\}$. Hence the solution of system (3) exists and unique.

Theorem 1 Assume that condition $w_{7} \geq w_{4}$ holds and let $\Lambda$ be the set defined by

$$
\begin{array}{r}
\Lambda=\left[(u, v, w) \in R_{+}^{3}: 0 \leq u \leq 1,0 \leq u+\frac{v}{w_{6}} \leq v_{c},\right. \\
\left.0 \leq u+\frac{v}{w_{6}}+\delta w \leq w_{c}\right],
\end{array}
$$

where $\delta=\frac{1}{w_{6} w_{11}}, p=\min \left\{w_{5}, w_{10}\right\}$ and $v_{c}=\left(1+\frac{1}{4 w_{5}}\right)$, $w_{c}=\left(1+\frac{1}{p}\right)_{6}$, then

(i) $\Lambda$ is positive invariant.

(ii) All positive solution of temporal system (3) is uniformly bounded and as time varies the critical solution eventually converges to the region $\Lambda$.

(iii) The temporal model (3) is dissipative.

Theorem 1 confirms the boundedness and dissipativeness of the system (3). The proof of this theorem is given in "Appendix 1".
Now, to justify the feasibility and existence of our proposed food chain model, system (3) need to satisfy the Kolmogorov sufficient and necessary conditions (May 1973). Kolmogorov system ensures periodic oscillations or chaotic dynamics in 3D system if two subsystem of any 3D system is K-system. Let us consider the reduced subsystem of temporal model system (3), we have

$$
\left\{\begin{array}{l}
\frac{\mathrm{d} u}{\mathrm{~d} t}=u\left((1-u)-\frac{v}{u+w_{4}}\right)=u F(u, v), \\
\frac{\mathrm{d} v}{\mathrm{~d} t}=v\left(-w_{5}+\frac{w_{6} u}{u+w_{7}}\right)=v G(u, v) .
\end{array}\right.
$$

In the two species dynamical models, the Kolmogorov theorem assures the existence of a stable limit cycle or stable equilibria and ensures biologically relevant parametric values for the model. Therefore, for the biologically relevant predator-prey model, we assume that the subsystem (4) should qualify as K-system. The subsystem (4) contemplate a K-system with the conditions:

$0<\frac{w_{5} w_{7}}{w_{6}-w_{5}}<1, w_{5}<w_{6}$

Moreover, the Kolmogorov system (4) contains three positive equilibrium points. The sustainability of these equilibria are summed as:

1. The equilibrium $E_{00}=(0,0)$ is exists with eigenvalues $1,-w_{5}$. Thus, the nature of the eigenvalue is characterized as saddle point.

2. The equilibrium $E_{10}=(1,0)$ always exist and is stable and eigenvalues are $-1,-w_{5}$

3. The equilibrium $E_{20}=(\tilde{u}, \tilde{v})$, where 
$\left\{\begin{array}{l}\tilde{u}=\frac{w_{5} w_{7}}{w_{6}-w_{5}} \\ \tilde{v}=(1-\tilde{u})\left(\tilde{u}+w_{4}\right)\end{array}\right.$

always exist and if

$2\left(\frac{w_{5} w_{7}}{w_{6}-w_{5}}\right)+w_{4}-1>0$

holds then $E_{20}$ is locally asymptotically stable.

\subsection{Stability of Temporal System}

In this subsection, we obtain existence conditions for equilibrium points of the temporal model (3) and the stability for positive equilibria $E_{3}=\left(u^{\star}, v^{\star}, w^{\star}\right)$ is investigated.

\subsubsection{Stability and Hopf Bifurcation Analysis}

The equilibrium points $E_{0}(0,0,0)$ and $E_{1}(1,0,0)$ always exist for model (3). The equilibrium point $E_{2}(\tilde{u}, \tilde{v}, 0)$ exist in $u-v$ plane, where $\tilde{u}$ and $\tilde{v}$ are given in Eq. (6). Further, due to deficiency of secondary consumer $(v)$ there is no equilibria exists in the $v-w$ plane.

Now to study the existence of equilibrium point $E_{3}=\left(u^{\star}, v^{\star}, w^{\star}\right)$, we consider following algebraic nonlinear equations:

$$
\left\{\begin{aligned}
g_{1}(u, v, w) & =1-u-\frac{v}{u+w_{4}}=0 \\
g_{2}(u, v, w) & =-w_{5}+\frac{w_{6} u}{u+w_{7}}-\frac{w}{v+w_{8} w+w_{9}} \\
& =0, \\
g_{3}(u, v, w) & =-w_{10}+\frac{w_{11} v}{v+w_{8} w+w_{9}}=0
\end{aligned}\right.
$$

Thus, solving the system (8), we get

$$
\left\{\begin{array}{l}
v^{\star}=\left(1-u^{\star}\right)\left(u^{\star}+w_{4}\right), \\
w^{\star}=\frac{\left(w_{11}-w_{10}\right) v^{\star}-w_{9} w_{10}}{w_{9} w_{10}} .
\end{array}\right.
$$

For $v^{\star}>0$ and $w^{\star}>0$ it follows that

$0<u^{\star}<1,0<\frac{w_{9} w_{10}}{w_{11}-w_{10}}<v^{\star}$.

Next, we explore the dynamical behavior of the model (3) via local stability around the feasible equilibrium points. The calculated variational matrix of the system at the point $(u, v, w)$ is rendered as:
$V(u, v, w)=\left(\begin{array}{ccc}u \frac{\partial g_{1}}{\partial u}+g_{1} & u \frac{\partial g_{1}}{\partial v} & u \frac{\partial g_{1}}{\partial w} \\ v \frac{\partial g_{2}}{\partial u} & v \frac{\partial g_{2}}{\partial v}+g_{2} & v \frac{\partial g_{2}}{\partial w} \\ w \frac{\partial g_{3}}{\partial u} & w \frac{\partial g_{3}}{\partial v} & w \frac{\partial g_{3}}{\partial w}+g_{3}\end{array}\right)$

$\frac{\partial g_{1}}{\partial u}=-1+\frac{v}{\left(u+w_{4}\right)^{2}}, \frac{\partial g_{1}}{\partial v}=-\frac{1}{\left(u+w_{4}\right)}$,

$\frac{\partial g_{1}}{\partial w}=0, \quad \frac{\partial g_{2}}{\partial u}=\frac{w_{6} w_{7}}{\left(u+w_{7}\right)^{2}}, \quad \frac{\partial g_{2}}{\partial v}=\frac{w}{\left(v+w_{8} w+w_{9}\right)^{2}}$,

$\frac{\partial g_{2}}{\partial w}=-\frac{v+w_{9}}{\left(v+w_{8} w+w_{9}\right)^{2}}, \quad \frac{\partial g_{3}}{\partial u}=0$,

$\frac{\partial g_{3}}{\partial v}=\frac{w_{11}\left(w_{8} w+w_{9}\right)}{\left(v+w_{8} w+w_{9}\right)^{2}}, \quad \frac{\partial g_{3}}{\partial w}=-\frac{w_{8} w_{11} v}{\left(v+w_{8} w+w_{9}\right)^{2}}$.

The linear stability around the critical points $E_{i}, i=$ $0,1,2,3$ are distinguished as:

(i) The equilibrium $E_{0}$ is saddle point and eigenvalues are given by $1,-w_{5},-w_{10}$.

(ii) The equilibrium point $E_{1}$ exist and eigenvalues are given by $-1,\left(-w_{5}+\frac{w_{6}}{1+w_{7}}\right), w_{10}$. It is stable if $w_{6}<w_{5}\left(1+w_{7}\right)$ and unstable if $w_{6}>w_{5}\left(1+w_{7}\right)$.

(iii) The root of characteristic equation $p_{3}(\lambda)=0$ of the above variation matrix (11) about $E_{2}=$ $(\tilde{u}, \tilde{v}, 0)$ satisfy the following condition:

$$
\begin{aligned}
& \lambda_{1}+\lambda_{2}=\tilde{u}\left[-1+\left(\frac{\tilde{v}}{\left(\tilde{u}+w_{4}\right)^{2}}\right)\right], \\
& \lambda_{1} \lambda_{2}=\frac{w_{6} w_{7} \tilde{u} \tilde{v}}{\left(\tilde{u}+w_{4}\right)\left(\tilde{u}+w_{7}\right)^{2}}, \\
& \lambda_{3}=\frac{w_{11} \tilde{v}}{\left(\tilde{v}+w_{9}\right)}-w_{10} .
\end{aligned}
$$

The equilibrium point $E_{2}=(\tilde{u}, \tilde{v}, 0)$ is stable or unstable in the positive $w$-direction contingents on parameter $w_{10}$ and $w_{11}$. If $\frac{w_{11} \tilde{v}}{\left(\tilde{v}+w_{9}\right)}<w_{10}$, then $E_{2}$ rests on being stable.

(iv) At $E^{\star}$, variational matrix $V\left(u^{\star}, v^{\star}, w^{\star}\right)$ reforms to

$V\left(u^{\star}, v^{\star}, w^{\star}\right)=\left(a_{i j}\right)_{3 \times 3}, \quad i, j=1,2,3$,

where 


$$
\begin{aligned}
& a_{11}=u^{\star}\left[-1+\left(\frac{v^{\star}}{\left(u^{\star}+w_{4}\right)^{2}}\right)\right], \\
& a_{12}=-\frac{u^{\star}}{\left(u^{\star}+w_{4}\right)}<0, a_{13}=0, \\
& a_{21}=\frac{w_{6} w_{7} v^{\star}}{\left(u^{\star}+w_{7}\right)^{2}}>0, \\
& a_{22}=\frac{v^{\star} w^{\star}}{\left(v^{\star}+w_{8} w^{\star}+w_{9}\right)^{2}}>0, \\
& a_{23}=-\frac{v^{\star}\left(v^{\star}+w_{9}\right)}{\left(v^{\star}+w_{8} w^{\star}+w_{9}\right)^{2}}<0, \\
& a_{31}=0, a_{32}=\frac{w_{11} w^{\star}\left(w_{8} w^{\star}+w_{9}\right)}{\left(v^{\star}+w_{8} w^{\star}+w_{9}\right)^{2}}, \\
& a_{33}=-\frac{w_{8} w_{11} v^{\star} w^{\star}}{\left(v^{\star}+w_{8} w^{\star}+w_{9}\right)^{2}} .
\end{aligned}
$$

Then the characteristic equation of (12) is

$\lambda^{3}+A_{1} \lambda^{2}+A_{2} \lambda+A_{3}=0$,

where

$$
\begin{aligned}
& A_{1}=-\left(a_{11}+a_{22}+a_{33}\right), \\
& A_{2}=\left(\left(a_{11}+a_{22}\right) a_{33}\right)-\left(a_{12} a_{21}-a_{11} a_{22}\right) \\
&-a_{23} a_{32}, \\
& A_{3}=\left(a_{12} a_{21}-a_{11} a_{22}\right) a_{33}+a_{11} a_{23} a_{32}, \\
& A_{1} A_{2}-A_{3}=\left(a_{11}^{2}-a_{32} a_{23}\right)\left(-a_{22}-a_{33}\right) \\
&+\left(a_{33}^{2}-a_{12} a_{21}\right)\left(-a_{11}-a_{22}\right) \\
&+a_{22}^{2}\left(-a_{11}-a_{33}\right)-2 a_{11} a_{22} a_{33} .
\end{aligned}
$$

Therefore, from Routh-Hurwitz criterion if

$a_{11}<0,\left(a_{11}+a_{22}\right)<0$,

$\left(a_{12} a_{21}-a_{11} a_{22}\right)<0$,

then the coefficients of Eq. (13) is strictly positive with $A_{1} A_{2}-A_{3}>0$. Hence, the coexisting equilibria $E_{3}\left(u^{\star}, v^{\star}, w^{\star}\right)$ is locally asymptotically stable.

Theorem 2 The condition for the happening of a Hopf bifurcation for system (3) at $w_{8}=\overline{w_{8}}$ are following

(i) $A_{i}\left(\overline{w_{8}}\right)>0, i=1,2,3$,

(ii) $A_{1}\left(\overline{w_{8}}\right) A_{2}\left(\overline{w_{8}}\right)-A_{3}\left(\overline{w_{8}}\right)=0$,

(iii) $\operatorname{Re}\left[\frac{d \lambda\left(w_{8}\right)}{\mathrm{d} w_{8}}\right]_{w_{8}=\overline{w_{8}}} \neq 0$.

Proof From the condition $A_{1} A_{2}-A_{3}$, we have

$$
\begin{aligned}
A_{1} A_{2}-A_{3}= & \left(a_{11}^{2}-a_{32} a_{23}\right)\left(-a_{22}-a_{33}\right) \\
& +\left(a_{33}^{2}-a_{12} a_{21}\right)\left(-a_{11}-a_{22}\right) \\
& +a_{22}^{2}\left(-a_{11}-a_{33}\right)-2 a_{11} a_{22} a_{33}=0 .
\end{aligned}
$$

Since $A_{2}>0$ at $w_{8}=\bar{w}_{8}$, there exist an interval containing $w_{8} \in\left(\overline{w_{8}}-\epsilon, \overline{w_{8}}+\epsilon\right)$, where $\epsilon>0$. Therefore for the interval $w_{8} \in\left(\overline{w_{8}}-\epsilon, \overline{w_{8}}+\epsilon\right)$ the characteristic equation (13) cannot have real roots contain negative real part. For $w_{8}=\overline{w_{8}}$, Eq. (13) can be written as

$$
\left(\lambda^{2}+A_{2}\right)\left(\lambda+A_{1}\right)=0 \text {, }
$$

which has exactly three roots $\lambda_{1}=i \sqrt{A_{2}}, \lambda_{2}=-i \sqrt{A_{2}}$ and $\lambda_{3}=-A_{1}$. Let

$$
\begin{aligned}
& \lambda_{1}\left(w_{8}\right)=\alpha\left(w_{8}\right)+i \beta\left(w_{8}\right), \\
& \lambda_{2}\left(w_{8}\right)=\alpha\left(w_{8}\right)-i \beta\left(w_{8}\right), \\
& \lambda_{3}\left(w_{8}\right)=-A_{1}\left(w_{8}\right) .
\end{aligned}
$$

Now for transversality condition, substituting above given value in Eq. (13), we get

$$
\left\{\begin{array}{l}
\Phi_{1}\left(w_{8}\right) \alpha^{\prime}\left(w_{8}\right)-\Phi_{2}\left(w_{8}\right) \beta^{\prime}\left(w_{8}\right)+M_{1}\left(w_{8}\right)=0 \\
\Phi_{2}\left(w_{8}\right) \alpha^{\prime}\left(w_{8}\right)+\Phi_{1}\left(w_{8}\right) \beta^{\prime}\left(w_{8}\right)+M_{2}\left(w_{8}\right)=0
\end{array}\right.
$$

where

$$
\begin{aligned}
\Phi_{1}\left(w_{8}\right) & =3 \alpha\left(w_{8}\right)+2 A_{1}\left(w_{8}\right) \alpha\left(w_{8}\right)+A_{2}\left(w_{8}\right)-3 \beta^{2}\left(w_{8}\right), \\
\Phi_{2}\left(w_{8}\right) & =6 \alpha\left(w_{8}\right) \beta\left(w_{8}\right)+2 A_{1}\left(w_{8}\right) \beta\left(w_{8}\right), \\
M_{1}\left(w_{8}\right) & =\alpha^{2}\left(w_{8}\right) A_{1}^{\prime}\left(w_{8}\right)+A_{2}^{\prime}\left(w_{8}\right) \alpha\left(w_{8}\right)+A_{3}^{\prime}\left(w_{8}\right) \\
& -A_{1}^{\prime}\left(w_{8}\right) \beta^{2}\left(w_{8}\right), \\
M_{2}\left(w_{8}\right) & =2 \alpha\left(w_{8}\right) \beta\left(w_{8}\right) A_{1}^{\prime}\left(w_{8}\right)+A_{2}^{\prime}\left(w_{8}\right) \beta\left(w_{8}\right) .
\end{aligned}
$$

Now solving Eq. (16), we get

$$
\operatorname{Re}\left[\frac{d \lambda_{j}}{\mathrm{~d} w_{8}}\right]_{w_{8}=w_{8}}=-\frac{\Phi_{1} M_{1}+\Phi_{2} M_{2}}{\Phi_{1}^{2}+\Phi_{2}^{2}} \neq 0,
$$

where $\Phi_{1} M_{1}+\Phi_{2} M_{2} \neq 0$ and $\lambda_{3}=-A_{1}\left(w_{8}\right)$. Thus, the transversality condition $\operatorname{Re}\left[\frac{d \lambda_{j}}{d a}\right]_{w_{8}=\overline{w_{8}}} \neq 0$ holds implying the rise of a Hopf bifurcation at $w_{8}=\overline{w_{8}}$.

In the next theorem, we analyze the sustainability of equilibria $E_{3}^{*}$ irrespective of initial conditions in the domain $\Lambda$.

Theorem 3 Under the conditions:

$$
\begin{aligned}
& v^{\star}<w_{4}\left(u^{\star}+w_{4}\right), \\
& w_{6} u^{\star}<w_{5}\left(u^{\star}+w_{7}\right), \\
& w_{6} w_{11} v_{c}\left(v^{\star}+w_{8}\right)<w_{9} w_{10}\left(v^{\star}+w_{8} w^{\star}+w_{9}\right), \\
& w_{8}^{2} w_{c}^{2}<\delta^{2} w_{9}\left(v^{\star}+w_{9}\right),
\end{aligned}
$$


equilibrium point $E_{3}^{\star}$ is globally asymptotically stable in the interior of the positive octant $\Lambda$ and the set $\Lambda$ is defined in Theorem 1.

The proof of Theorem 3 is conceded in "Appendix 2".

\section{Analysis of Spatio-Temporal System}

\subsection{Stability of Spatial Model}

Here, we analyze the effect of diffusion for the spatially homogeneous equilibrium $E_{3}\left(u^{\star}, v^{\star}, w^{\star}\right)$ for the model system (2). The interaction among the prey $u(x, y, t)$, secondary consumer $v(x, y, t)$ and tertiary consumer $w(x, y, t)$ is described in system (2).

To study the spatial dynamics of model system (2), we linearize the model about the equilibrium $E_{3}\left(u^{\star}, v^{\star}, w^{\star}\right)$ as follows

$$
\left\{\begin{array}{l}
\frac{\partial \bar{u}}{\partial t}=a_{11} \bar{u}+a_{12} \bar{v}+a_{13} \bar{w}+d_{1} \nabla^{2} \bar{u} \\
\frac{\partial \bar{v}}{\partial t}=a_{21} \bar{u}+a_{22} \bar{v}+a_{23} \bar{w}+d_{2} \nabla^{2} \bar{v} \\
\frac{\partial \bar{w}}{\partial t}=a_{31} \bar{u}+a_{32} \bar{v}+a_{33} \bar{w}+d_{3} \nabla^{2} \bar{w}
\end{array}\right.
$$

where we introduce small perturbations $\bar{u}=u-u^{\star}, \bar{v}=v-v^{\star}, \bar{w}=w-w^{\star}$. Let the solution of the system (22) is of the form

$$
\left(\begin{array}{c}
\bar{u} \\
\bar{v} \\
\bar{w}
\end{array}\right)=\left(\begin{array}{c}
c_{1} \\
c_{2} \\
c_{3}
\end{array}\right) \exp \left(\lambda_{k} t\right) \cos \left(k_{x} x\right) \cos \left(k_{y} y\right) .
$$

Here $c_{i}, i=1,2,3$ are constants. $k_{x}$ and $k_{y}$ are the components of wave number along Cartesian plane $(x, y)$ with wave length $\lambda_{k}$.

The variational matrix of the linearized model system is given by Eq. (22) is defined as

$$
\bar{J}=\left(\begin{array}{ccc}
a_{11}-d_{1} k^{2} & a_{12} & a_{13} \\
a_{21} & a_{22}-d_{2} k^{2} & a_{23} \\
a_{31} & a_{32} & a_{33}-d_{3} k^{2}
\end{array}\right),
$$

where $k$ is the wave number given by $k^{2}=k_{x}^{2}+k_{y}^{2}$ and characteristic equation of (24) is given by

$\lambda_{k}^{3}+\rho_{1} \lambda_{k}^{2}+\rho_{2} \lambda_{k}+\rho_{3}=0$,

where

$$
\begin{aligned}
\rho_{1}= & \left(d_{1}+d_{2}+d_{3}\right) k^{2}+A_{1}, \\
\rho_{2}= & \left(d_{1} d_{2}+d_{2} d_{3}+d_{1} d_{3}\right) k^{4}-\left[d_{1}\left(a_{22}+a_{33}\right)\right. \\
& +d_{2}\left(a_{11}+a_{33}\right)+d_{3}\left(a_{11}+a_{22}\right] k^{2}+A_{2}, \\
\rho_{3}= & d_{1} d_{2} d_{3} k^{6}-\left[a_{11} d_{2} d_{3}+a_{22} d_{1} d_{3}+a_{33} d_{1} d_{2}\right] k^{4} \\
& +\left[d_{1}\left(a_{22} a_{33}-a_{23} a_{32}\right)+d_{2} a_{11} a_{33}\right. \\
& \left.+d_{3}\left(a_{11} a_{22}-a_{12} a_{21}\right)\right] k^{2}+A_{3},
\end{aligned}
$$

with $A_{1}, A_{2}$ and $A_{3}$ are defined in Eq. (13).

Theorem 4 Uniform steady state $E^{*}$ of system (2) is locally asymptotically stable if following conditions satisfy:

(i) $\quad \rho_{i}\left(k^{2}\right)>0$ where $i=1,2,3$.

(ii) $\rho_{1}\left(k^{2}\right) \rho_{2}\left(k^{2}\right)-\rho_{3}\left(k^{2}\right)>0$.

The proof of this theorem is simple and determined by the Routh-Hurwitz criterion. Hence, the proof is omitted here.

Theorem 5 The globally asymptotically stable steady state of the temporal model is also globally stable for the corresponding spatial model.

This theorem states that if the temporal system (3) is globally asymptotically stable around the positive equilibrium $E_{3}=\left(u^{*}, v^{*}, w^{*}\right)$, then corresponding spatial system (2) is also globally asymptotically stable in presence of diffusion. Theorem 5 verification is remitted in "Appendix 3".

\subsection{Turing Instability}

This subsection deals with the derivation of conditions that ensure the occurrence of Turing instability of the model system (2). Turing instability occurs when the stable equilibrium point of the temporal system becomes unstable by adding diffusion. It may occur for a certain range of wave number with an authentic choice of parameter values. However, The Turing instability conditions need not be determined by the geometry of the model system, it determined by the reaction rates and diffusion coefficients (Baek et al. 2013). Turing instability may occur if at least one root of Eq. (25) has the positive real part for some $k \geq 0$. Disregarding the sign of $\rho_{1}$ and $\rho_{2}$ in Eq. (25), the equation has a positive real root if $\rho_{3}=q\left(k^{2}\right) \leq 0$. Therefore, diffusion driven instability occurs when $q\left(k^{2}\right) \leq 0$. Hence condition for diffusion instability is given by

$q\left(k^{2}\right)=p_{0} k^{6}+p_{1} k^{4}+p_{2} k^{2}+A_{3}$,

where 


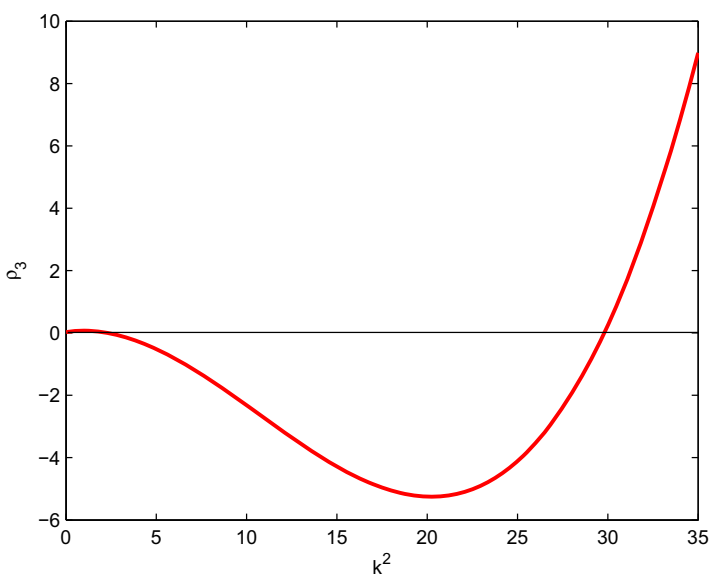

(a)

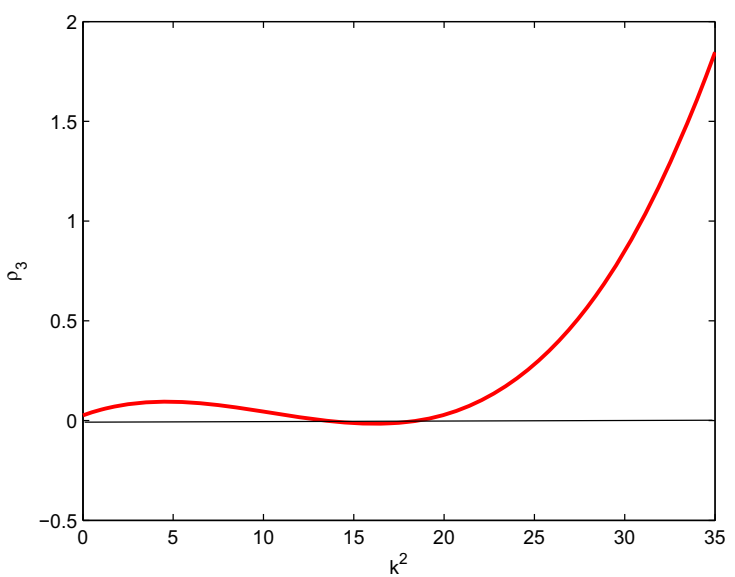

(b)

Fig. 1 Turing instability occurs at negative $\rho_{3}$ for some $k$ with diffusion coefficients a $d_{1}=1, d_{2}=0.01, d_{3}=0.01, \mathbf{b} d_{1}=0.5, d_{2}=0.05$, $d_{3}=0.06$, and rest of parameters are defined on Eq. (26)

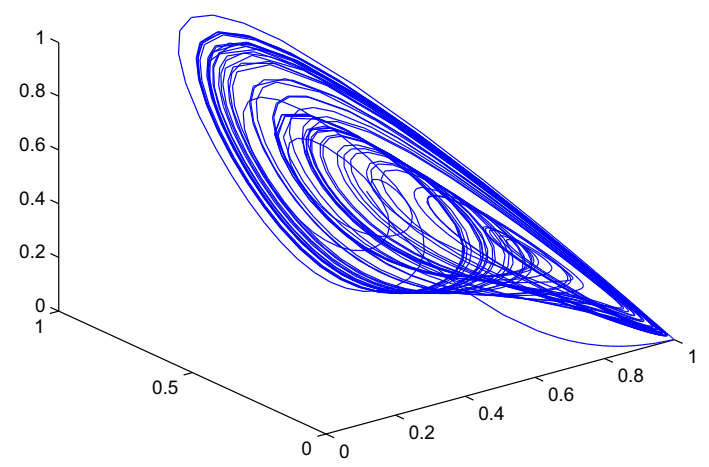

(a)

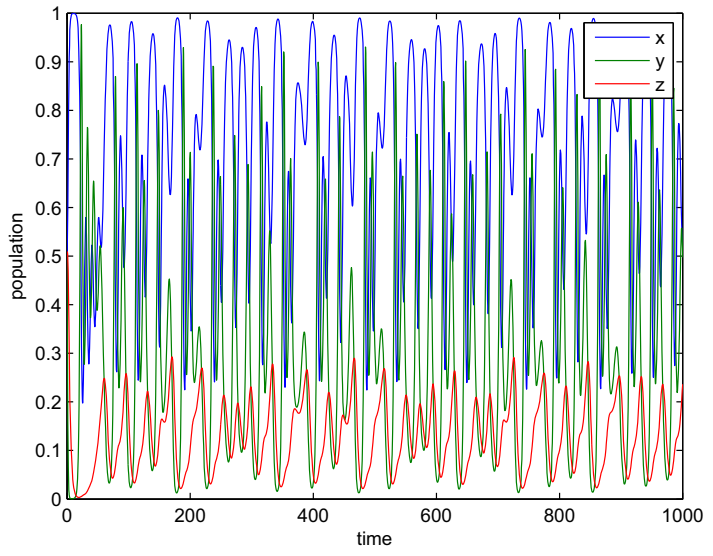

(b)

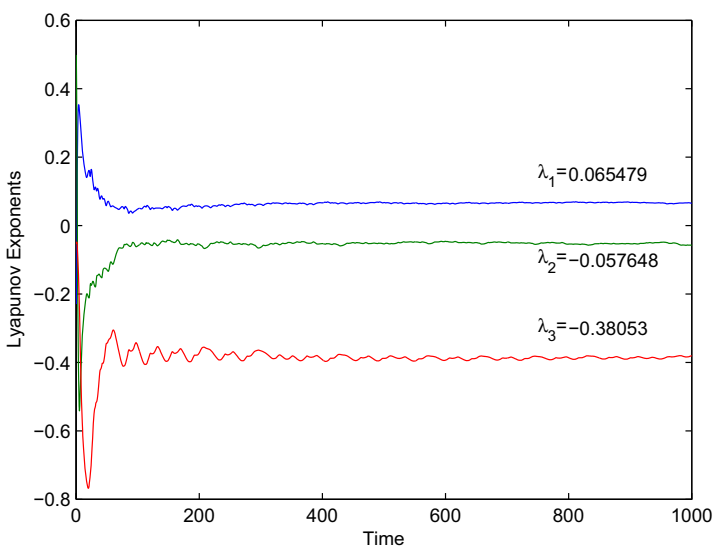

(c)

Fig. 2 a Chaotic attractor for model (3) at $w_{9}=0.08$, b time series for chaos, c time versus Lyapunov exponent 


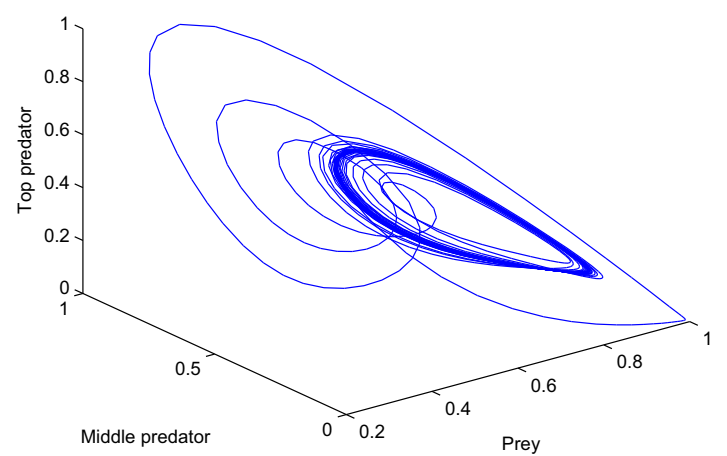

(a)

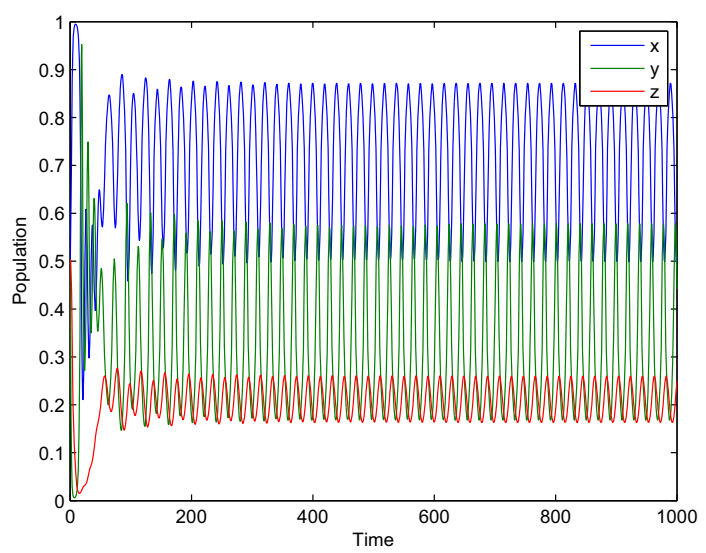

(b)

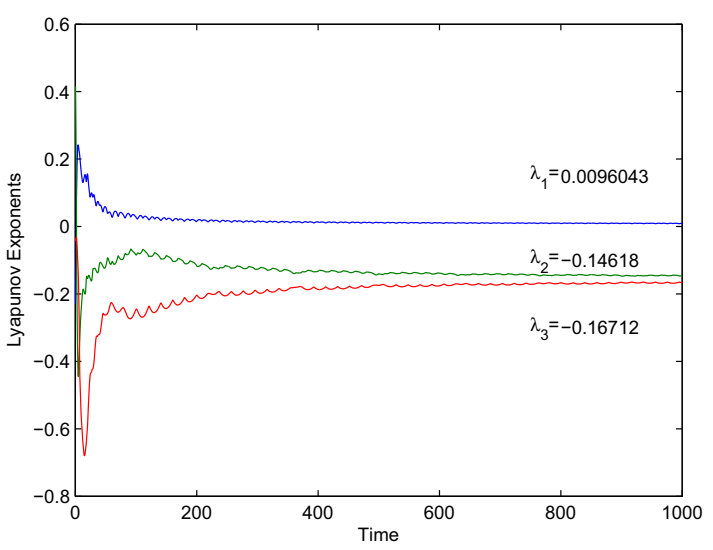

(c)

Fig. 3 a Limit cycle for model (3) at $w_{9}=0.12$, b time series of species population for limit cycle, c time versus Lyapunov exponent

$$
\begin{aligned}
p_{0}= & d_{1} d_{2} d_{3}, \\
p_{1}= & -\left(a_{11} d_{2} d_{3}+a_{22} d_{1} d_{3}+a_{33} d_{1} d_{2}\right), \\
p_{2}= & d_{1}\left(a_{22} a_{33}-a_{23} a_{32}\right)+d_{2} a_{11} a_{33} \\
& +d_{3}\left(a_{11} a_{22}-a_{12} a_{21}\right) .
\end{aligned}
$$

The minimum value of $q\left(k^{2}\right)$ occur at $k=k_{c r}$ given by

$k_{c r}=\sqrt{\frac{\left(-p_{1}+\sqrt{p_{1}^{2}-3 p_{0} p_{2}}\right)}{3 p_{1}}}$.

Straight forward algebraic calculations show at least one root of Eq. (25) has positive real part, and thus diffusive instability occur if either $p_{2}<0$ or $p_{0}<0$ and $p_{1}^{2}>3 p_{0} p_{2}$. However, Turing instability does not not exist for model (2) if condition (14) hold.

We have discussed the Turing instability conditions of the model system (2) for a particular set parameter values with diffusion coefficients. For example, the following parameter values

$$
\left\{\begin{array}{c}
w_{4}=0.5, w_{5}=1.15, w_{6}=2.93, w_{7}=0.58, \\
w_{8}=0.21, w_{9}=0.16, w_{10}=0.35, w_{11}=0.55,
\end{array}\right.
$$

the conditions $\rho_{1}(0)=0.3251>0, \rho_{2}(0)=0.2093>0$, $\rho_{3}=0.0264>0$ and

$\rho_{1}(0) \rho_{2}(0)-\rho_{3}(0)=0.04164434>0$,

thus $E_{3}\left(u^{\star}, v^{\star}, w^{\star}\right)$ is locally stable. Further, in presence of diffusion $d_{1}=1, d_{2}=0.01, d_{3}=0.01, \rho_{3}$ changes its sign with any positive wave number $k>0$ as shown in Fig. 1.

\section{Numerical Simulations}

\subsection{Numerical Simulation of Temporal System}

In this subsection, we venture to support our analytical findings numerically via MATLAB programming. Also, we perceive the diverse and complex dynamics of the model system in this section. These observations are 


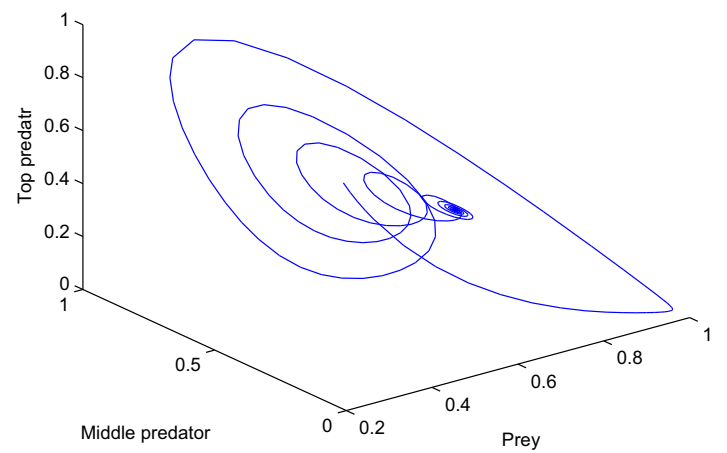

(a)

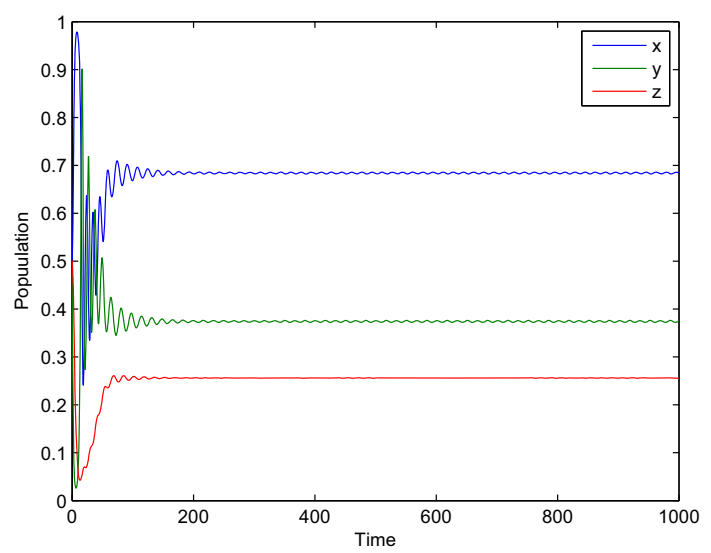

(b)

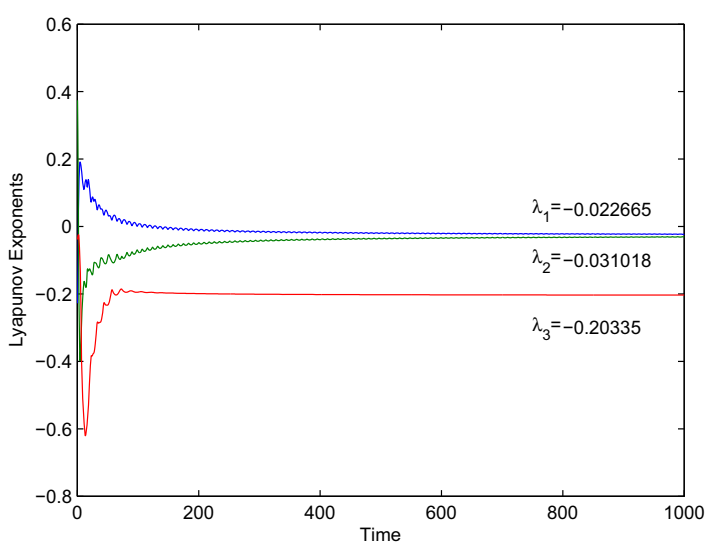

(c)

Fig. 4 a Stable focus for model (3) at $w_{9}=0.16$, b time evolution showing stability, c time versus Lyapunov exponent

inspected via phase portraits, time evolution, and Lyapunov exponent. It is observed that for parameter values given in Eq. (26) of model system (3) shows distinct attractors for the parameter value of $w_{9}$ as given in Figs. 2, 3,4 . We fix biological relevant parameter values defined in (26) for numerical experiments. We obtain positive equilibrium point as $E_{5}\left(u^{\star}, v^{\star}, w^{\star}\right)=(0.684,0.374,0.255)$ for parameter values defined in Eq. (26). We testify that the model (3) manifests the global behavior for this parameter set.

The model system (3) shows chaotic behaviour at $w_{9}=0.08$, simulated in the Fig. 2a with time evolution of species populations in Fig. 2b. The Lyapunov exponent for this attractor is calculated as $0.065479,-0.057648$, and -0.38053 accumulated in Fig. 2c. Here the appearance of both positive and negative Lyapunov exponent reinforces the existence of chaos for the model system (3). We have simulated the periodic behavior at the parameter value $w_{9}=0.12$ for the model system (3) in Fig. 3. We have ascertained the Lyapunov exponent for this attractor as
0.0096048 , -0.14618 , and -0.16712 , and the convergence of the first Lyapunov exponent toward 0 validates the periodic behavior of the model system (3).

As we increase the parameter value of $w_{9}$, the system emergence towards stability, and finally at $w_{9}=0.16$, we witness the stable focus dynamics for the temporal model as given in Fig. 4a, b. Negative Lyapunov exponents $-0.022655,-0.031018$, and -0.20335 as simulated in Fig. 4c authenticates this argument. The tertiary consumer free equilibrium point $E_{2}$ with condition (6) is calculated as $u=0.375, v=0.547, w=0$, as presented in Fig. 5 .

\subsubsection{Bifurcation Analysis}

In this section, bifurcation analysis is performed for the non-spatial model. All the bifurcation diagrams are plotted for the parameter values given in Eq. (26). The bifurcation diagrams show intricate and rich dynamics representing various sequences of period-doubling and period-halving bifurcation leading to limit cycles and chaos. Also, we can 


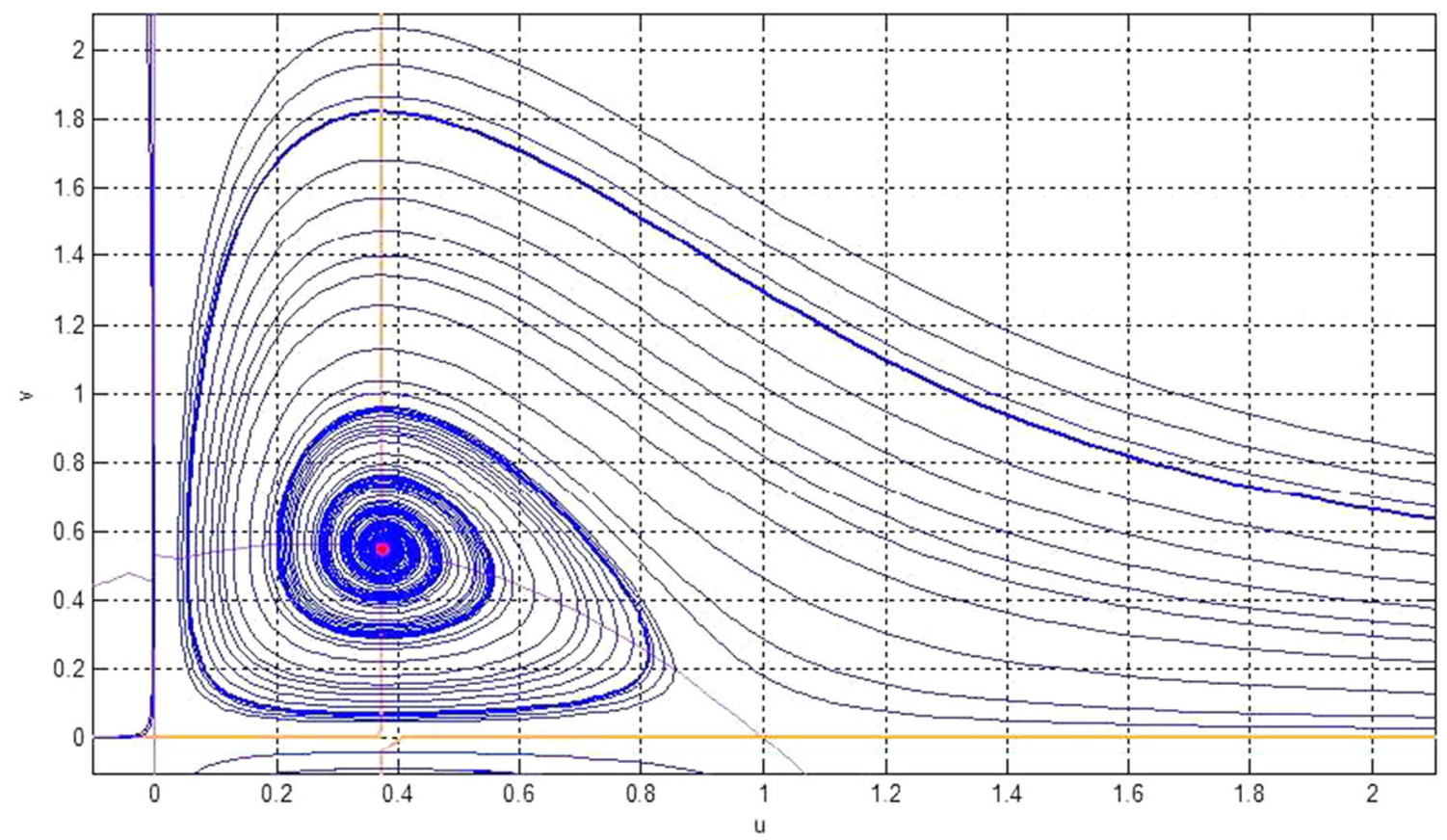

Fig. 5 Equilibrium point $E_{2}=(0.375,0.547,0)$

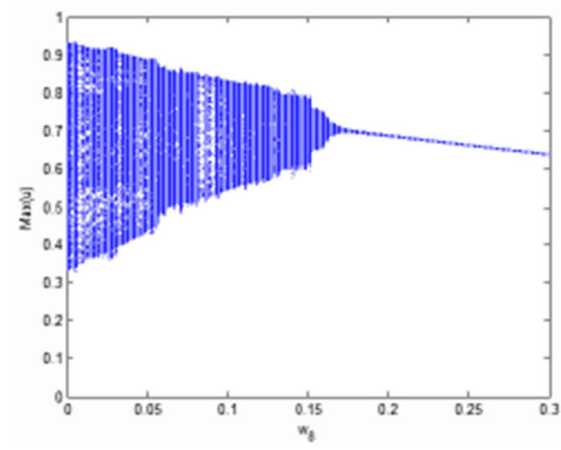

(a)

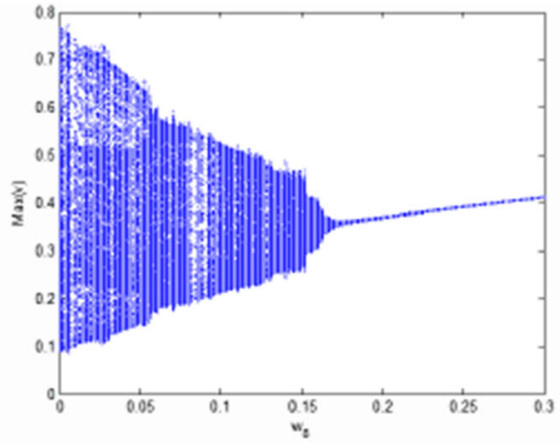

(b)

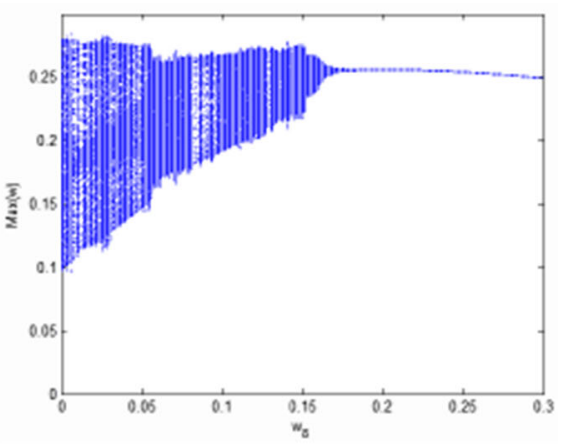

(c)

Fig. 6 Bifurcation diagrams corresponding to parameter $w_{8}$ for the temporal system (3)

verify the phase portrait dynamics by bifurcation diagrams corresponding to the parameter. In Fig. 6, we present bifurcation diagram for successive maxima of $u, v, w$ in the ranges $[0.0,1.0],[0.0,0.8]$ and $[0.0,0.3]$, respectively as a function of $w_{8}$ in the range $0.0 \leq w_{8} \leq 0.3$. In Fig. 7, the successive maxima of $u, v, w$ is given for the variation of parameter value $w_{10}$ in the range $0.2 \leq w_{10} \leq 0.4$. In Fig. 8, the successive variations of prey $(u)$, consumer $(v)$ and consumer $(w)$ are taken concerning the parameter $w_{11}$ in the interval $0.5 \leq w_{11} \leq 0.75$.

\subsection{Numerical Simulation for Spatial Model}

In this section, we have found inclusive results for the spatial model (2). We have assumed the following set of parameters for the numerical solution of the spatial model system (2):

$w_{4}=0.5, w_{5}=1.3, w_{6}=2.93, w_{7}=0.58$,

$w_{8}=0.21, w_{9}=0.16, w_{10}=0.35$,

$w_{11}=0.55, d_{1}=1, d_{2}=0.01, d_{3}=0.01$.

\subsubsection{One Dimensional Simulation}

This subsection deals with the simulation of the system (2) with one dimensional Laplacian operator $\nabla^{2}=\frac{\partial^{2}}{\partial x^{2}}$. Here, the populations $u(x, 0)>0, v(x, 0)>0$ and $w(x, 0)>0$, $x \in[0, R]$, and zero flux boundary conditions are given by 


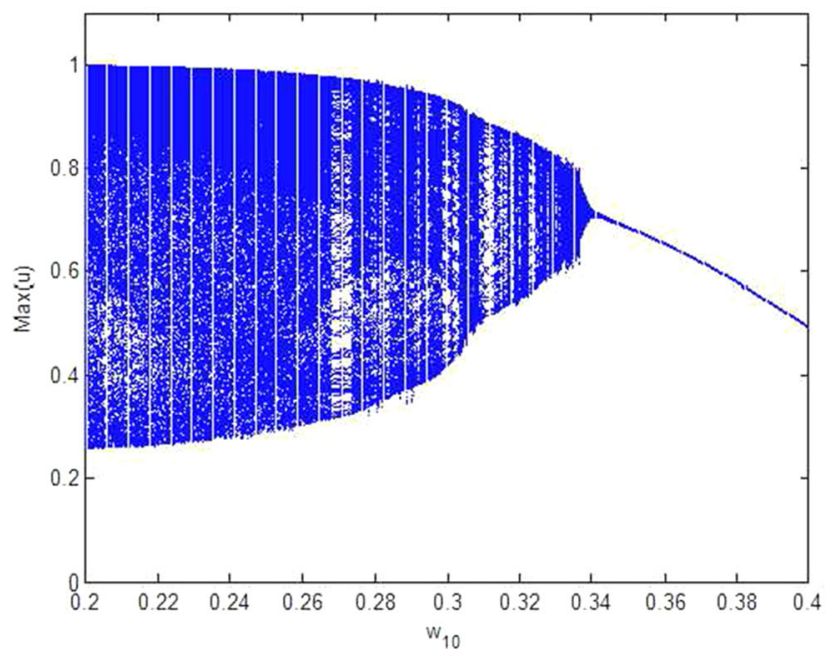

(a)

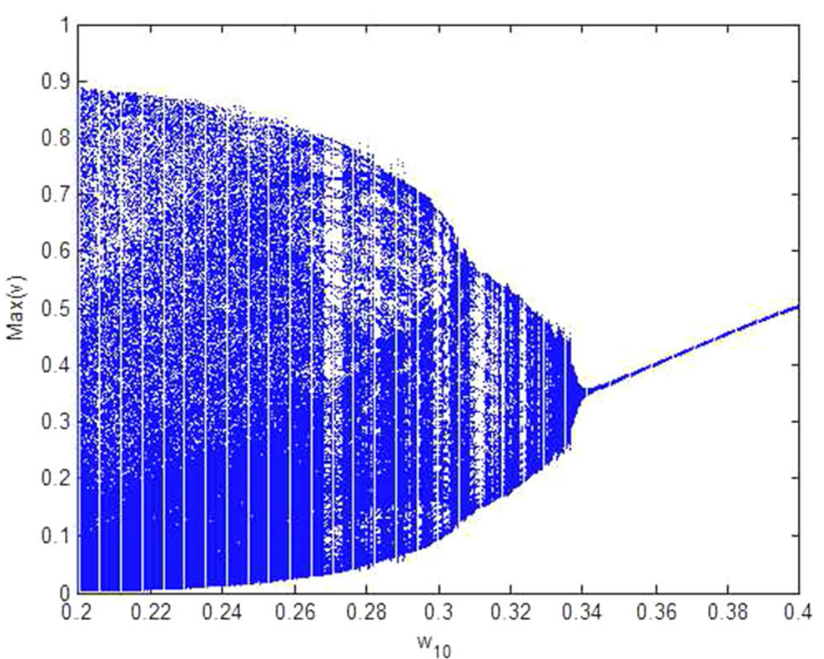

(b)

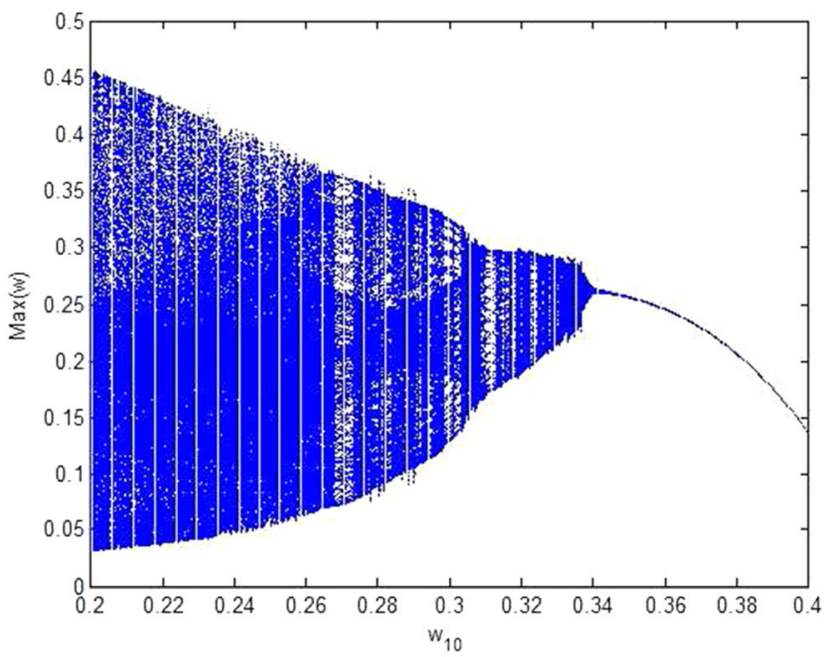

(c)

Fig. 7 Bifurcation diagrams corresponding to parameter $w_{10}$ for the temporal system (3)

$\frac{\partial u}{\partial x}=\frac{\partial v}{\partial x}=\frac{\partial w}{\partial x}=0$.

We have plotted space versus population density to scrutinize the spatial dynamics. Here, we have considered the non-monotonic initial conditions as

$$
\begin{aligned}
& u(x, 0)=u^{\star}+\epsilon\left(u-x_{1}\right)\left(u-x_{2}\right), \\
& v(x, 0)=v^{\star}, \quad w(x, 0)=w^{\star},
\end{aligned}
$$

where $\left(u^{\star}, v^{\star}, w^{\star}\right)$ is steady state for the coexistence of primary consumer (prey), secondary consumer and tertiary consumer populations and $\epsilon=10^{-8}, x_{1}=1200, x_{2}=2800$ is the parameter affecting the model dynamics. We have studied the behavior of model system (2) in 1D space for the set of parameter values given in Eq. (27).
Spatial dynamics of model (2) is given in the form of phase portrait diagram for different value of $w_{8}$ in Fig. 10. We obtain the spatial dynamics of the model (27) varying time. System is stable at time $t=10$ but as we increase time, there are onset chaotic faces at $t=500$ and $t=5000$. In Fig. 9, the population density of prey and predator shows some jagged patterns representing the chaotic behavior. This phenomenon is known as Wave of Chaos (see Petrovskii and Malchow 2014; Upadhyay et al. 2009, 2016) in spatio-temporal system.

\subsubsection{Two-Dimensional Simulation}

This section deals with the simulation of the spatial system in two-dimensional space. We study spatio-temporal complexity with the help of pattern formulation. 


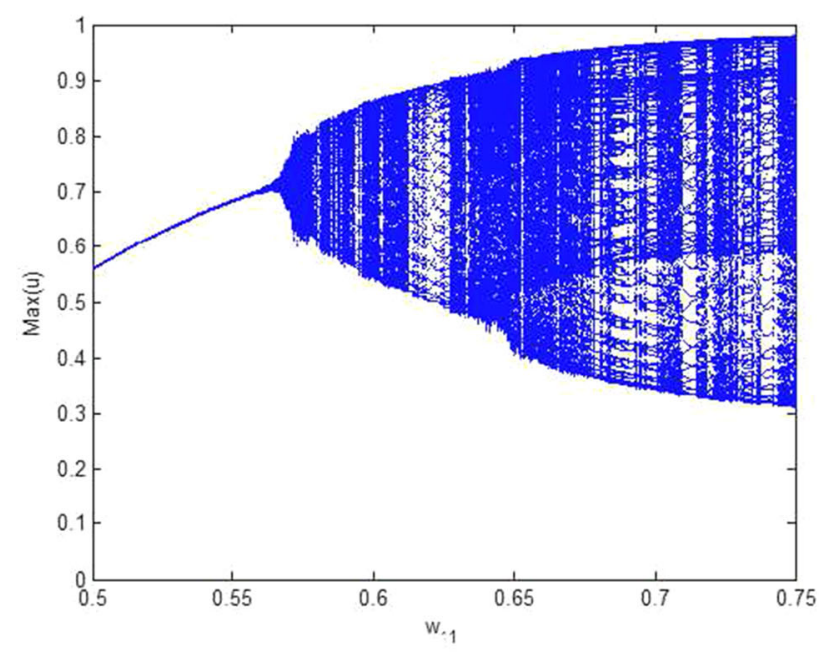

(a)

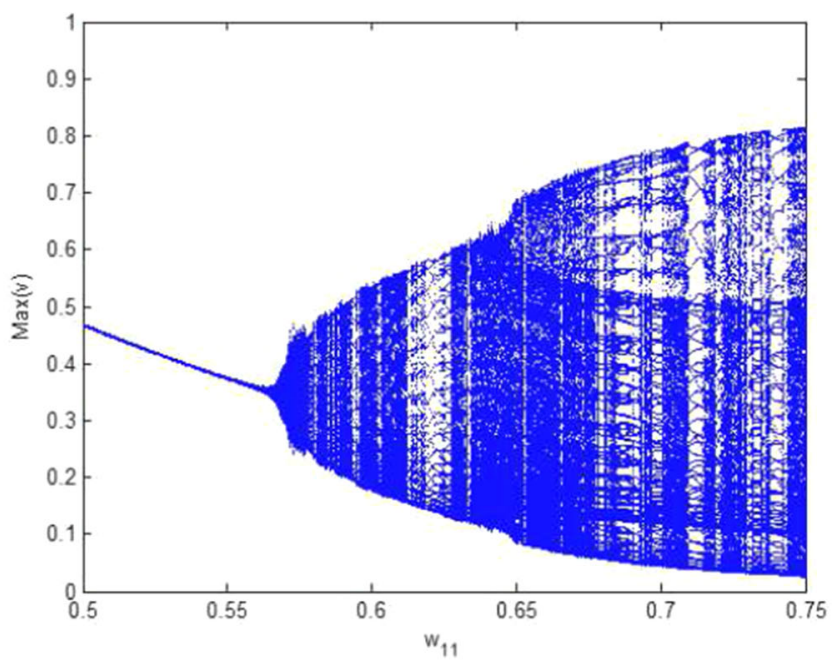

(b)

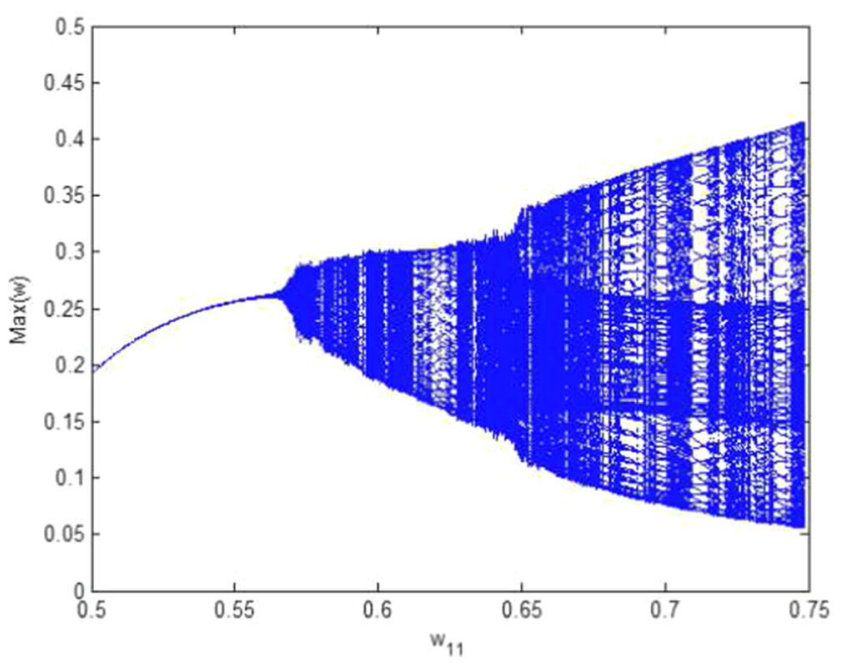

(c)

Fig. 8 Bifurcation diagrams corresponding to parameter $w_{11}$ for the temporal system (3)

We have adopted the forward difference scheme to solve the reaction part and the standard five points explicit finite difference scheme method for the two-dimensional diffusion term. Further, we have exerted nonzero initial and zero-flux boundary conditions with the system size of $50 \times 50$. Also, we can implement Euler methods to determine time evaluations. Here, the values of the time and space for simulation of the spatial model system is considered as $\Delta h=0.25, \Delta t=0.001$. The dynamics of the spatiotemporal system depends upon the choice of initial conditions (Medvinsky et al. 2002). The initial distribution of the species for two-dimensional simulation is considered as

$$
\begin{gathered}
u(x, y, 0)=u^{\star}+0.1 \cos ^{2}(10 x) \cos ^{2}(10 y), \\
v(x, y, 0)=v^{\star}+0.1 \cos ^{2}(10 x) \cos ^{2}(10 y), \\
w(x, y, 0)=w^{\star}+0.1 \cos ^{2}(10 x) \cos ^{2}(10 y),
\end{gathered}
$$

about steady state.

Now, we present the evolutionary process of Turing pattern formulation and complex spatial patterns for the two different set of parameter values. Snapshots of the spatial distribution are shown for different time intervals $t=500,5000,10,000$ in Fig. 11 . We plot the contour spatial distributions for population $u, v, w$ at different time intervals with $50 \times 50$ points for $d_{1}=1, d_{2}=0.001, d_{3}=$ 0.01 and rest parameter values defined in Eq. (27). In Fig. 11a, we observe stripe patterns with emergence of interference patterns in the left lower boundary of domain. 


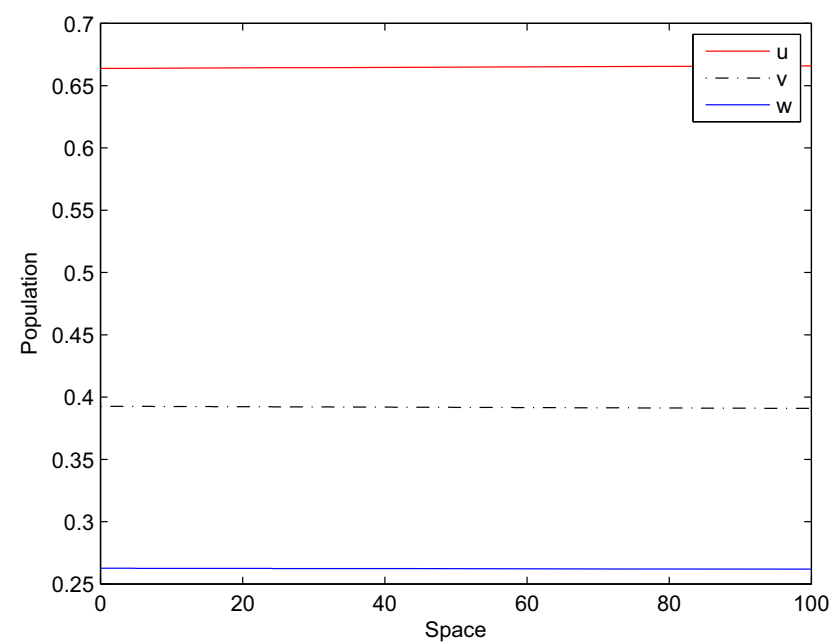

(a)

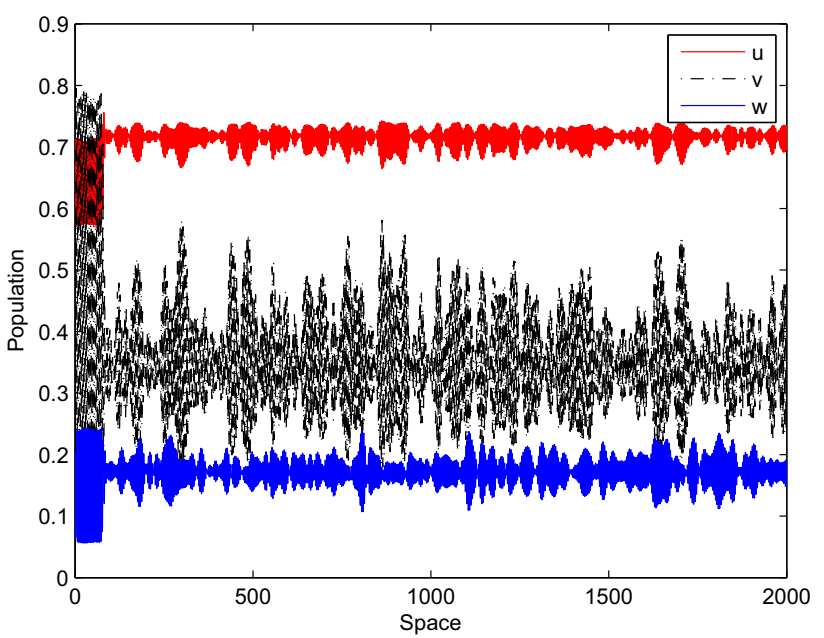

(b)

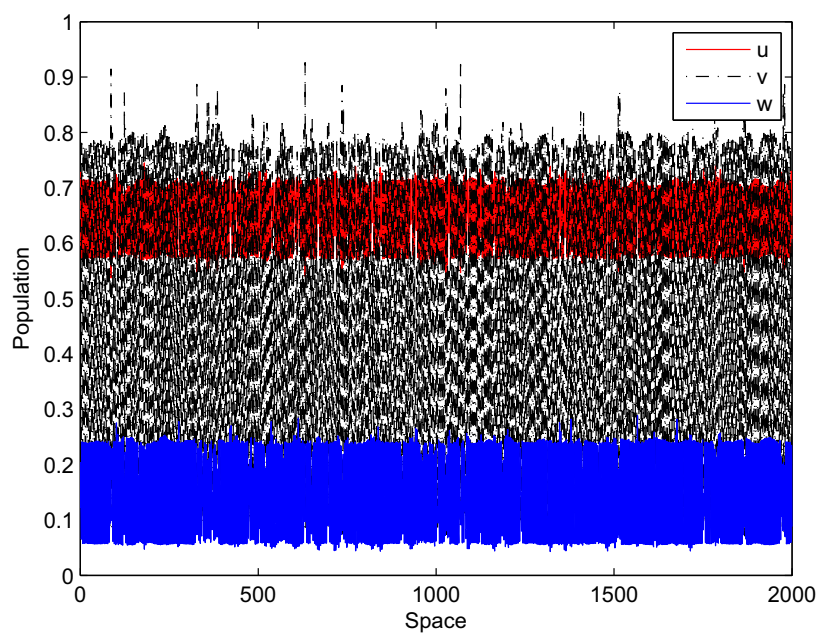

(c)

Fig. 9 One dimensional (1D) space series for spatial model (2) at time $\mathbf{a} t=10, \mathbf{b} t=500, \mathbf{c} t=2000$ and all other parameter values are given in Eq. (27)

A close examination on these patterns suggest that as time increases, the spatial distribution changes from one pattern to another chaotically and we get very complex patterns. At $t=5000$, mixture of stripe and interference patterns are observed, although interference pattern dominate in this case (see Fig. 11b). Finally, at time $t=10,000$, we see interference patterns dominating in the whole domain (see Fig. 11c).

From Figs. 12, 13, we observe that pattern of spatial distribution depends upon mutual interference parameter $w_{8}$. Here the blue spot signifies the patches at low population density and the red spot exposes the patches at high population density. Also, these patterns are named as spot patterns in literature (see Malchow 1993; Medvinsky et al. 2002). Increasing the value of $w_{8}$ in Hopf-Turing domain effect in spatial distribution and it can be seen that complex spot (or 'leopard') patterns changed into the mixture of spot and stripe pattern.

\section{Discussion and Conclusion}

If food is abundant the predator does not interfere with each other but a striking competition occurs between predators for food while the scarcity of prey species happens. The main purpose of this work is to detect the complex dynamics in the tri-tropic food chain model when mutual interference is adopted by the predators during the predation. We incorporate Bedington-DeAngelis functional as a predation function between the predators. The 


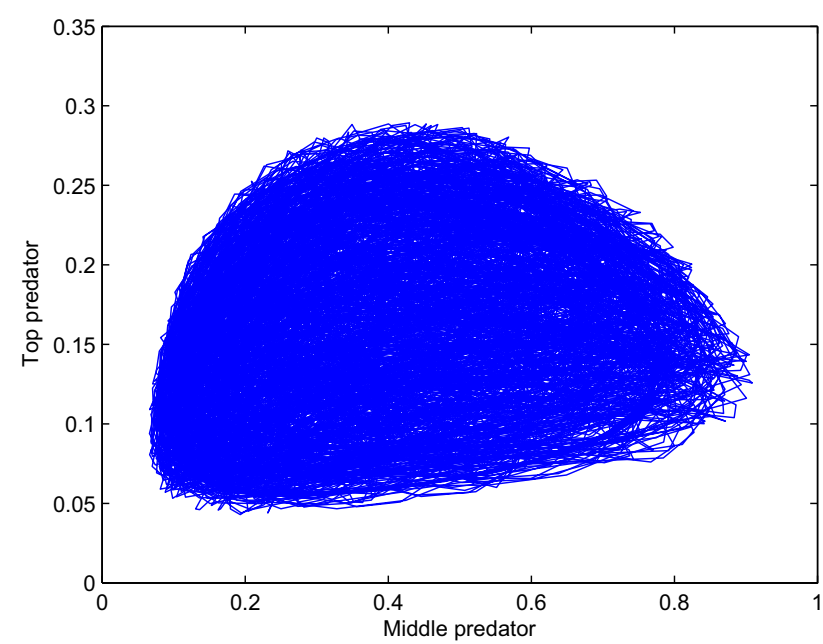

(a)

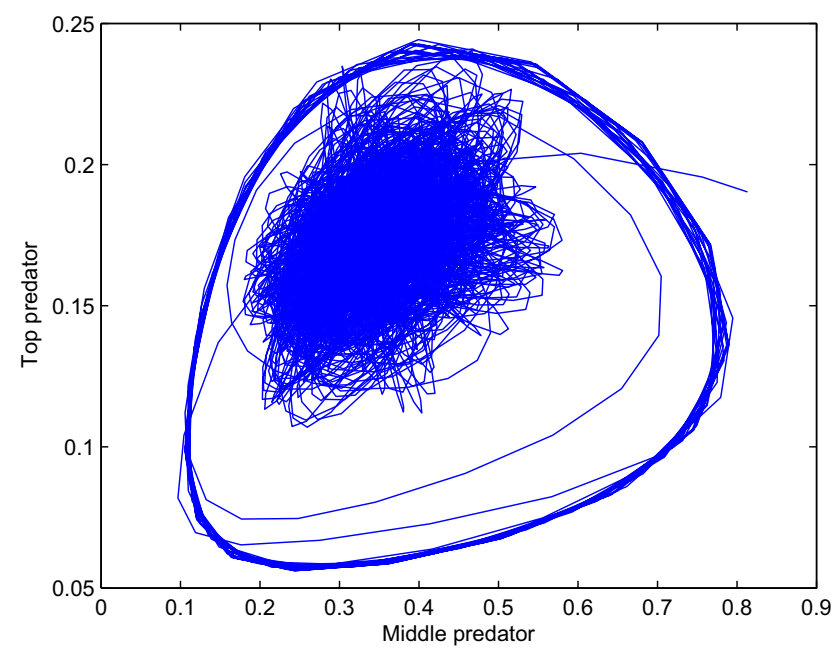

(c)

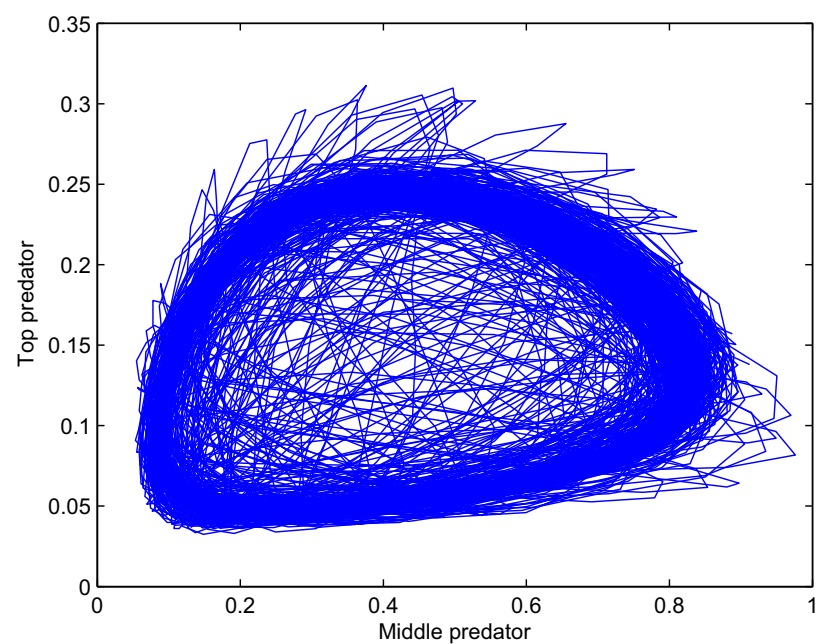

(b)

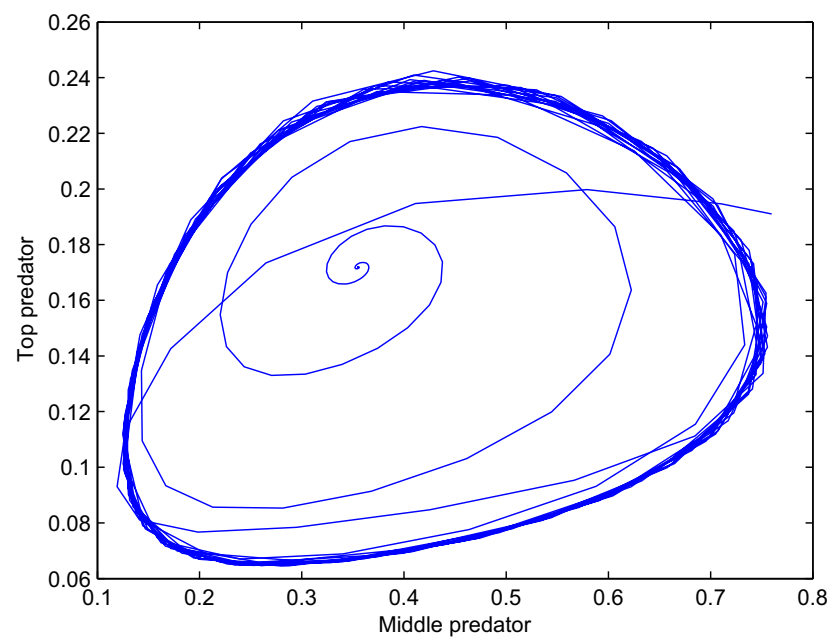

(d)

Fig. 10 Phase portrait of spatial model (2) for $\mathbf{a} w_{8}=0.15, \mathbf{b} w_{8}=0.2, \mathbf{c} w_{8}=0.25, \mathbf{d} w_{8}=0.3$ at $t=5000$ and all other parameter values are given in Eq. (27)

dynamics of the non-spatial model system have been studied with the help of phase portrait, time evolution, and bifurcation diagram. Several studies have been investigated respecting the predator-prey model with BeddingtonDeAngelis functional response. The research exposes the diverse dynamics including species extinction, persistence, stable or unstable equilibria, limit cycles, and strange attractors (commonly known as chaos) (see Baghel and Dhar 2014; Dobromir and Dimitrov 2005; DeAngelis et al. 1975; Hwang 2003; Naji and Balasim 2007; Upadhyay et al. 2013). Boundedness and Kolmogorov conditions are obtained for non-spatial model. Figure 4 shows the locally asymptotically positive equilibria $E_{3}$ for model (3). We have simulated the equilibria $E_{2}$ in Fig. 5 and the coordinate values where the $\mathrm{u}$-nullclines and $\mathrm{v}$-nullclines intersect is calculated as $u=0.375, v=0.547$ and $w=0$. Also, this confirms the stability of equilibrium point $E_{20}$ for the
Kolmogorov system (4). We observe the non-spatial model goes for Hopf bifurcation at some critical value of the interference parameter. Bifurcation diagrams show the consequences of mutual interference and other important parameters of the predator-prey model. The study shows that mutual interference plays a central role in the dynamics of the predator-prey system. It is observed that a large value of interference parameter $\left(w_{8}\right)$ ensures stability, but leads to a fall in the top predator population. In Fig. 6, this result is simulated and for $w_{8} \geq 0.17$, the system is approaching toward stability. Furthermore, the interference parameter $\left(w_{8}\right)$ has also the ability to stabilize the spatial model (2) and Fig. 10 witness this result.

It is seen that some individual among a group of prey changes location either in search of resources, food or to avoid the threat of predation. During this movement of prey species, predator senses the insufficiency of food and 

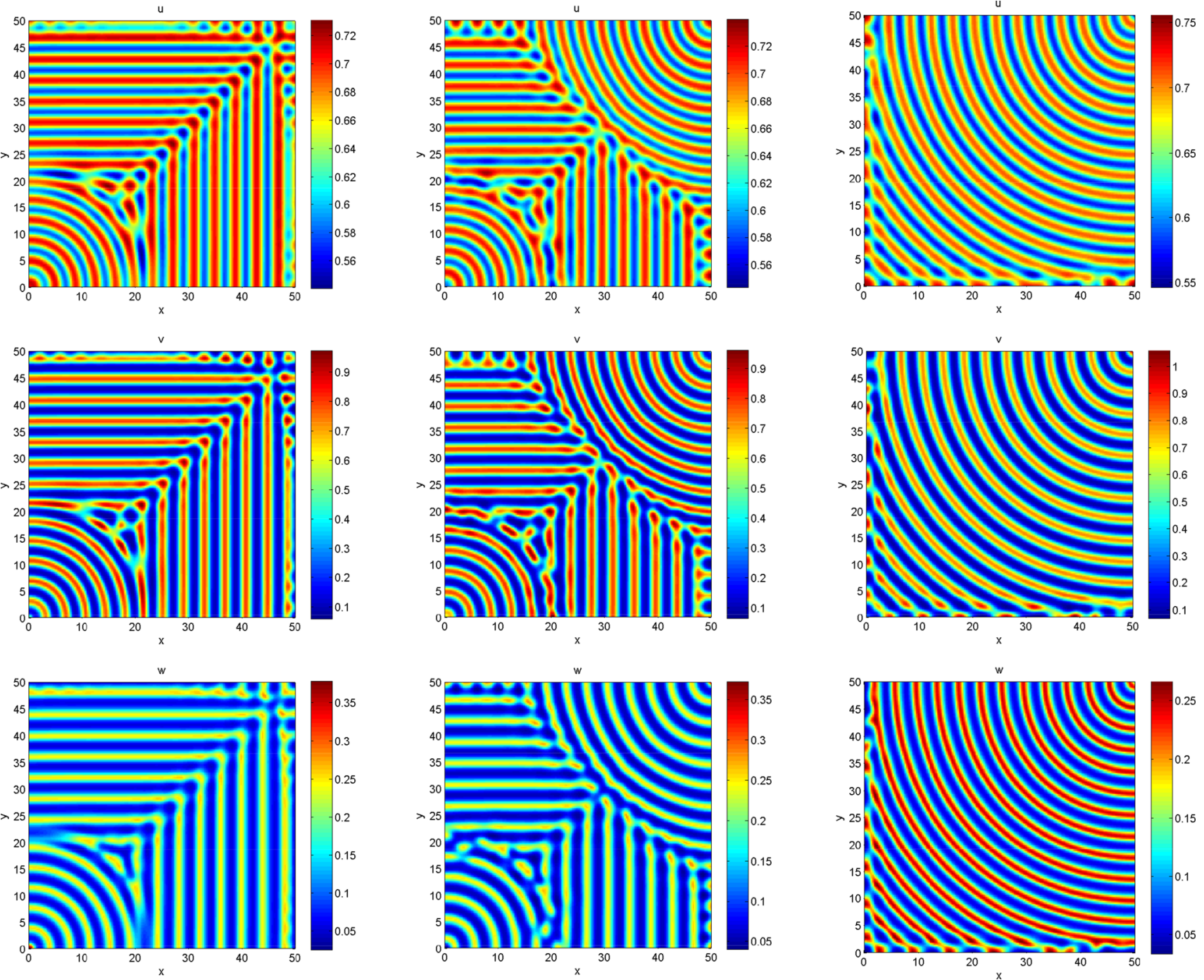

(a)

(b)

(c)

Fig. 11 Turing patterns represented by contour plot at $\mathbf{a} t=100, \mathbf{b} t=5000$, $\mathbf{c} t=10,000$ in two dimension $(x-y)$ plane and all other parameters defined in Eq. (27)

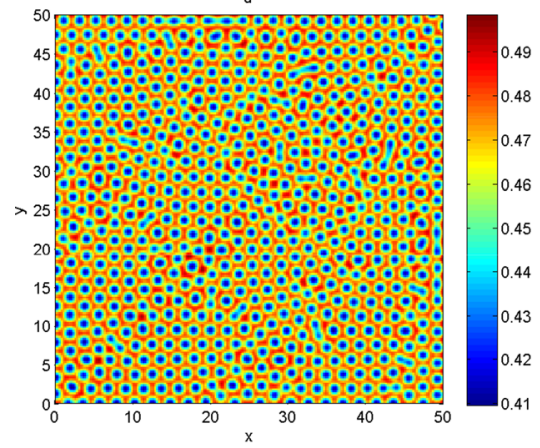

(a)

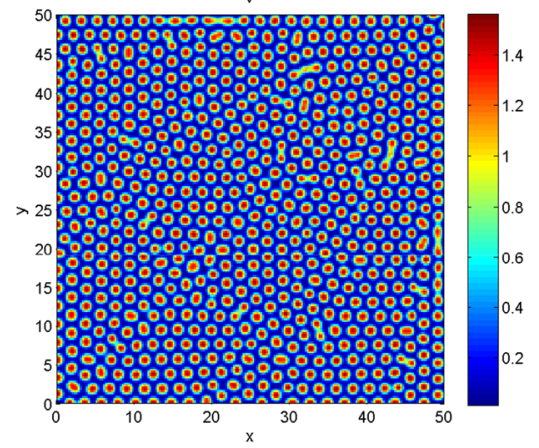

(b)

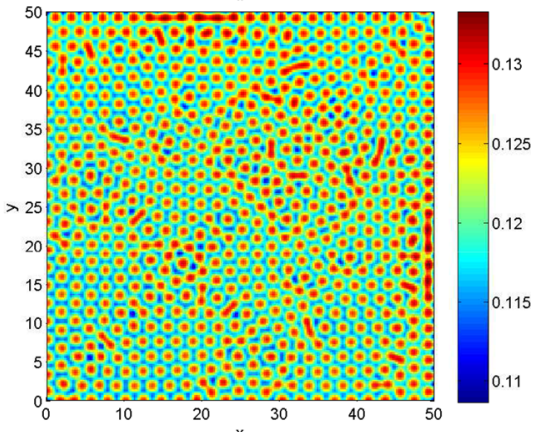

(c)

Fig. 12 Spatial distribution of species at the time $t=5000$ and $w_{8}=0.1$ in two dimension $(x-y)$ plane where parameter values are defined in Eq. (26) and diffusion coefficients are given by $d_{1}=1.5, d_{2}=0.01, d_{3}=0.01$ 


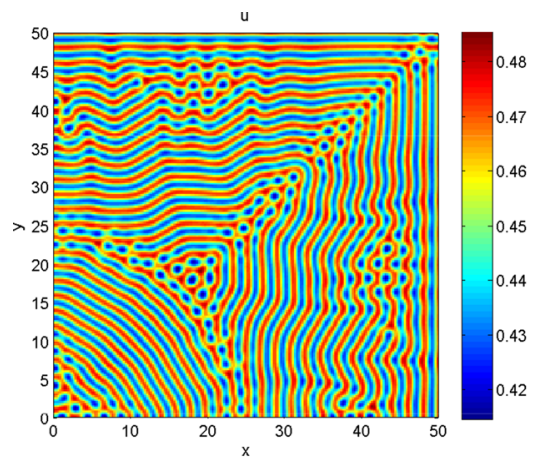

(a)

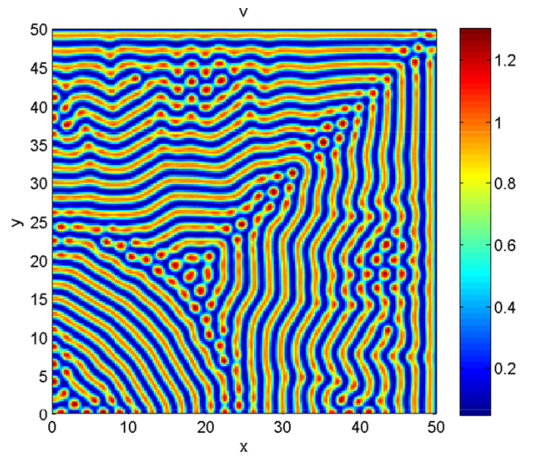

(b)

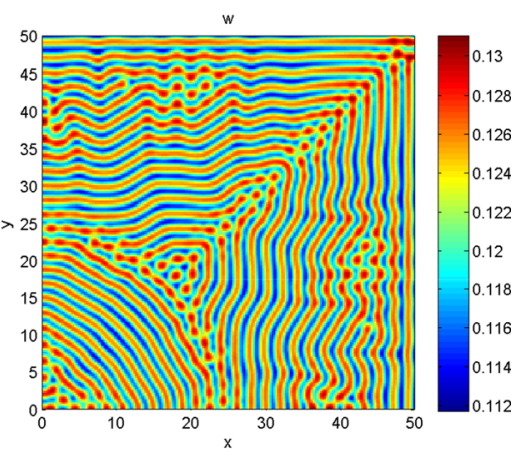

(c)

Fig. 13 Spatial distribution of species at the time $t=5000$ and $w_{8}=0.25$ in two dimension $(x-y)$ plane defined in Eq. (26) and diffusion coefficients are given by $d_{1}=1.5, d_{2}=0.01, d_{3}=0.01$

they also follow them toward the prey rich habitat. The biological conformity to these sequential movements of species produces structure distinguished as the travelling wave, modulated chaotic wave or chaotic behavior. Many different aspects like random dispersal of species, self prey growth, conversion rule, mutual interference and death rate of predators are considered. It is observed that these aspects of the reaction-diffusion predator-prey model impersonate the spatial structure for some period of interference during diffusion instabilities.

We observe the Turing patterns especially emphasizing on Beddington-DeAngelis functional response. A mixture of dominating interference pattern and stripe patterns has been observed and as time increases, one can see the emergence of the interference pattern (see Fig. 11). From the point of view of physics, if the light from two point sources overlaps, the interference pattern maps out how the phase difference between the two waves varies in space. Here this is determined by the separation of the point sources and the wavelength. An example of an interference pattern is a generation of Newton's ring pattern. We observe that patterns are strongly dependent on interference parameter $\left(w_{8}\right)$ and patterns change chaotically from one pattern to another. For a small value of $w_{8}=0.1$, hot and cold spot patterns can be seen. For instance when $w_{8}$ is varied from $w_{8}=0.1$ to $w_{8}=0.25$, mixture of spot with stripe patterns are observed in Figs. 12 and 13. Hot spot patterns are formed by the localized annular patches with a high concentration of population density of secondary and tertiary consumers. The appearance of such stripe (or 'zebra') patters is due to auto-catalysis saturation for high prey concentration values. Since prey can not increase further the region of high prey concentration become wider. The model system (2) encounters structural transitions and produces a variety of patterns (see Figs. 10, 11, 12, 13) for interference parameter and diffusion coefficients, indicating the significant importance of these parameters on pattern selection.

The idea contained in this paper gives an impeccable approach to examine how functional responses of species and parameters influence on the dynamics of a food chain model. We observe that the diffusion coefficients as well as mutual interference parameter between predators are responsible for the emergence of chaotic dynamics in the ecological model. In this study, we observe the emergence of complex patterns like spots, stripes and interference. This study shows that patterns in ecological interaction are an excellent tool to examine the fundamental mechanism of complexity that arises in a spatiotemporal model. From the study, we can conclude that if predators mutually interfere during predation, chaotic dynamics can automatically emerge in both temporal and spatial model.

\section{Appendix 1: Proof of Theorem 1}

Consider the following inequality from the system (3), we have

$$
\frac{\mathrm{d} u}{\mathrm{~d} t} \leq u(1-u)
$$

which implies $u(t) \leq\left(\frac{1}{G e^{-t}+1}\right)$, where $c=\left(\frac{1}{u_{0}}-1\right)$.
Hence, $\quad u(t) \leq 1, \forall t \geq 0$. $\sigma(t)=u(t)+\frac{v(t)}{w_{6}}$, we have

$$
\begin{aligned}
\frac{\mathrm{d} \sigma}{\mathrm{d} t} & =\frac{\mathrm{d} u}{\mathrm{~d} t}+\frac{1}{w_{6}} \frac{\mathrm{d} v}{\mathrm{~d} t}, \\
\Rightarrow \frac{\mathrm{d} \sigma}{\mathrm{d} t} & =u(1-u)-\frac{w_{5}}{w_{6}} v-u v\left(\frac{w_{7}-w_{4}}{\left(u+w_{4}\right)\left(u+w_{7}\right)}\right)
\end{aligned}
$$




$$
-\frac{v w}{w_{6}\left(v+w_{8} w+w_{9}\right)}
$$

Here, the solutions of linearized ODE system (3) are locally Lipschitz continuous functions. Then, if $w_{7} \geq w_{4}$, the equation (30) transforms to

$\frac{\mathrm{d} \sigma}{\mathrm{d} t} \leq u(1-u)-\frac{w_{5}}{w_{6}} v$,

holds for all positive $u$ and $v$. Further, Eq. (31) is converted to

$\frac{\mathrm{d} \sigma}{\mathrm{d} t}+w_{5} \sigma(t) \leq u(1-u)+w_{5} u$.

But, $\max \{u(1-u)\}=\frac{1}{4}$, hence we get

$\frac{\mathrm{d} \sigma}{\mathrm{d} t}+w_{5} \sigma(t) \leq \frac{1}{4}+w_{5}$

Now by using comparison lemma,

$$
\begin{aligned}
\sigma(t) \leq & \left(\frac{1}{4 w_{5}}+1\right)-\left[\left(1+\frac{1}{4 w_{5}}-\sigma(0)\right) e^{-w_{5} t}\right], \\
\sigma(t) \leq & \left(\frac{1}{4 w_{5}}+1\right) \\
& -\left[\left(1+\frac{1}{4 w_{5}}-\left\{u(0)+\frac{v(0)}{w_{6}}\right\}\right) e^{-w_{5} t}\right] .
\end{aligned}
$$

Thus, for all $t \geq 0$, we have

$\sigma(t) \leq \frac{1}{4 w_{5}}+1 \Rightarrow u(t)+\frac{v(t)}{w_{6}} \leq \frac{1}{4 w_{5}}+1$.

If $\eta(t)=u(t)+\frac{v(t)}{w_{6}}+\delta w(t)$ with $\delta=\frac{1}{w_{6} w_{11}}$, then for $w_{7} \geq w_{4}$, we get

$$
\begin{aligned}
& \frac{\mathrm{d} \eta}{\mathrm{d} t} \leq u-\frac{w_{5}}{w_{6}} v-w_{10} \delta w, \\
\Rightarrow & \frac{\mathrm{d} \eta}{\mathrm{d} t}+p \eta \leq(1+p),
\end{aligned}
$$

where $p=\min \left\{w_{5}, w_{10}\right\}$. By using comparison lemma, we obtain for all $t \geq \tilde{T} \geq 0$,

$\eta(t) \leq\left(1+\frac{1}{p}\right)-\left[\left(1+\frac{1}{p}\right)-\sigma(\tilde{T})\right] e^{-p(t-\tilde{T})}$.

If $\tilde{T}=0$, then

$$
\begin{aligned}
& \eta(t) \leq\left(1+\frac{1}{p}\right)-\left[\left(1+\frac{1}{p}\right)-\sigma(0)\right] e^{-p t}, \\
& \eta(t) \leq\left(1+\frac{1}{p}\right), \forall t \geq 0 .
\end{aligned}
$$

\section{Appendix 2: Proof of Theorem 3}

Let

$$
S=\left[u-u^{\star}-u^{\star} \log \left(\frac{u}{u^{\star}}\right)\right]+\frac{k_{1}}{2}\left(v-v^{\star}\right)^{2}+\frac{k_{2}}{2}\left(w-w^{\star}\right)^{2}
$$

is the considered Lyapunov function with constant $k_{1}$ and $k_{2}$ around the equilibria $E_{3}=\left(u^{*}, v^{*}, w^{*}\right)$ for the temporal system (3). Time derivative of Lyapunov function $S$ is

$$
\begin{aligned}
\frac{\mathrm{d} S}{\mathrm{~d} t}= & -\frac{1}{2} a_{11}\left(u-u^{\star}\right)^{2}+a_{12}\left(u-u^{\star}\right)\left(v-v^{\star}\right) \\
& -\frac{1}{2} a_{22}\left(v-v^{\star}\right)^{2}-\frac{1}{2} a_{22}\left(v-v^{\star}\right)^{2} \\
& +a_{23}\left(v-v^{\star}\right)\left(w-w^{\star}\right)-\frac{1}{2} a_{33}\left(w-w^{*}\right)^{2} \\
& -\frac{1}{2} a_{11}\left(u-u^{*}\right)^{2}+a_{13}\left(u-u^{*}\right)\left(w-w^{*}\right) \\
& -\frac{1}{2} a_{33}\left(w-w^{*}\right)^{2}
\end{aligned}
$$

where

$$
\begin{aligned}
a_{11} & =1-\frac{v^{\star}}{\left.\left(u+w_{4}\right)\right)\left(u^{\star}+w_{4}\right)}, \\
a_{22} & =k_{1}\left(w_{5}-\frac{w_{6} u^{\star}}{\left(u^{\star}+w_{7}\right)}\right. \\
& \left.+\frac{w\left(w_{9}+w_{8} w\right)}{\left(v^{\star}+w_{8} w^{\star}+w_{9}\right)\left(v+w_{8} w+w_{9}\right)}\right) \\
a_{33} & =k_{2}\left(w_{10}-\frac{w_{11} v\left(v^{\star}+w_{8}\right)}{\alpha_{3}}\right) \\
a_{12} & =\left(-\frac{1}{u+w_{4}}+\frac{k_{1} w_{6} w_{7} v}{\alpha_{2}}\right) \\
a_{23} & =\left(\frac{k_{2} w_{11} w^{\star}\left(w_{8} w+w_{9}\right)}{\left(v^{\star}+w_{8} w^{\star}+w_{9}\right)\left(v+w_{8} w+w_{9}\right)}\right. \\
& \left.-\frac{k_{1} v^{\star}\left(v^{\star}+w_{9}\right)}{\left(v^{\star}+w_{8} w^{\star}+w_{9}\right)\left(v+w_{8} w+w_{9}\right)}\right), a_{13}=0 .
\end{aligned}
$$

$\frac{\mathrm{d} S}{\mathrm{~d} t}$ is negative definite, if the following inequalities holds:

$a_{11}>0, a_{22}>0, a_{33}>0$,

$a_{12}^{2}<a_{11} a_{22}$,

$a_{23}^{2}<a_{22} a_{33}$,

$a_{13}^{2}<a_{11} a_{33}$.

Since $a_{13}=0$, condition (37) is automatically satisfied. Under the condition (18), $a_{11}>0$ and for conditions (19) and (20), $a_{22}>0$ and $a_{33}>0$, respectively. If we choose

$$
\begin{array}{r}
k_{1}=\frac{u^{\star}+w_{7}}{\left(1+w_{4}\right)\left(w_{6}^{2} v_{c}\right)} \text { and, } \\
k_{2}=\frac{k_{1} w_{9} v^{\star}\left(v^{\star}+w_{9}\right)}{w_{11} w^{\star}\left(w_{8}\left(W_{c} / \delta\right) w_{9}\right)\left(v^{\star}+w_{8}\left(W_{c} \delta\right)+w_{9}\right.},
\end{array}
$$


then the conditions (35) and (36) are fulfilled. Hence, $E_{3}^{\star}$ is globally asymptotically stable.

\section{Appendix 3: Proof of Theorem 5}

Consider the function $E(t)$ as

$$
E(t)=\iint_{\Omega} S(u, v, w) \mathrm{d} A,
$$

where $S(u, v, w)$ is a Lyapunov function defined in Eq. (32).

For global stability of system (2), it is sufficient to show that $\frac{\mathrm{d} E}{\mathrm{~d} t} \leq 0$. Hence,

$\frac{\mathrm{d} E}{\mathrm{~d} t}=I_{1}+I_{2}$,

where

$I_{1}=\iint_{\Omega} \frac{\mathrm{d} S}{\mathrm{~d} t} \mathrm{~d} A$, and

$I_{2}=\iint_{\Omega}\left(d_{1} \frac{\partial S}{\partial u} \nabla^{2} u+d_{2} \frac{\partial S}{\partial v} \nabla^{2} v+d_{3} \frac{\partial S}{\partial w} \nabla^{2} w\right) \mathrm{d} A$.

Using Green's identity in the plane

$\iint_{\Omega}\left(F \nabla^{2} G\right) \mathrm{d} A=\int_{\partial \Omega} F \frac{\partial G}{\partial n} \mathrm{~d} s-\iint_{\Omega}(\nabla F \cdot \nabla G) \mathrm{d} A$.

Now, we have

$$
\begin{aligned}
& \iint_{\Omega}\left(d_{1} \frac{\partial S}{\partial u} \nabla^{2} u\right) \mathrm{d} A \\
& =-d_{1} \iint_{\Omega} \frac{\partial^{2} S}{\partial u^{2}}\left[\left(\frac{\partial u}{\partial x}\right)^{2}+\left(\frac{\partial u}{\partial y}\right)^{2}\right] \mathrm{d} A \leq 0, \\
& \iint_{\Omega}\left(d_{2} \frac{\partial S}{\partial v} \nabla^{2} v\right) \mathrm{d} A \\
& =-d_{2} \iint_{\Omega} \frac{\partial^{2} S}{\partial v^{2}}\left[\left(\frac{\partial v}{\partial x}\right)^{2}+\left(\frac{\partial v}{\partial y}\right)^{2}\right] \mathrm{d} A \leq 0, \\
& \iint_{\Omega}\left(d_{3} \frac{\partial S}{\partial w} \nabla^{2} w\right) \mathrm{d} A \\
& =-d_{3} \iint_{\Omega} \frac{\partial^{2} S}{\partial w^{2}}\left[\left(\frac{\partial w}{\partial x}\right)^{2}+\left(\frac{\partial w}{\partial y}\right)^{2}\right] \mathrm{d} A \leq 0 .
\end{aligned}
$$

From above analysis, it is clear that $I_{2} \leq 0$ and we have only to show $I_{1} \leq 0$ to prove $\frac{d E}{\mathrm{~d} t} \leq 0$. Hence it is proved that the globally stable equilibrium point $E_{3}=\left(u^{\star}, v^{\star}, w^{\star}\right)$ for system (3) will remain globally asymptotically stable for the diffusive system (2).

Acknowledgements This work is supported by ECR Award (File No. ECR/2017/000141) SERB-DST, New Delhi.

Author's contribution SNR: Supervision; conceptualization (lead); writing - original draft (lead); review and editing (equal); PM: methodology (lead); formal analysis (lead); BPS: investigation; writing - review and editing (equal); BT: writing - review and editing (equal).

\section{Declarations}

Conflict of interest The authors declare that they have no conflict of interest.

\section{References}

Ali KK, Osman MS, Baskonus HM, Elazabb NS, Ilhan E (2020) Analytical and numerical study of the HIV-1 infection of CD4+ T-cells conformable fractional mathematical model that causes acquired immunodeficiency syndrome with the effect of antiviral drug therapy. Math Methods Appl Sci 2020:1-17

Alonso D, Bartumeus F, Catalan J (2002) Mutual interference between predators can give rise to Turing spatial patterns. Ecology 83:28-34

Araujo SBL, de Aguiar MAM (2007) Pattern formation, outbreaks, and synchronization in food chains with two and three species. Phys Rev E 75:061908-061914

Baek H, Jung DI, Wang ZW (2013) Pattern formation in a semi ratiodependent predator-prey system with diffusion. Discrete Dyn Nat Soc 83:1-14

Baghel RS, Dhar J (2014) Pattern formulation in three species food web model in spatiotemporal domain with Beddington-DeAngelis functional response. Nonlinear Anal Model Control 19:155-171

Banerjee M, Abbas S (2015) Existence and non-existence of spatial patterns in a ratio-dependent predator-prey model. Ecol Complex 21:199-214

Banerjee M, Banerjee S (2012) Turing instability and spatio-temporal chaos in ratio dependence Holling-Tanner model. Math Biol 236:64-76

Banerjee M, Volpert V (2017) Spatio-temporal pattern formation in Rosenzweig-MacArthur model: effect of non- local interactions. Ecol Complex 30:2-10

Baskonus HM (2016) New acoustic wave behaviors to the DaveyStewartson equation with power-law nonlinearity arising in UID dynamics. Nonlinear Dyn 86:177-183

Baskonus HM (2019) Complex surfaces to the fractional $(2+1)$ dimensional Boussinesq dynamical model with the local M-derivative. Eur Phys J Plus 134:322

Beddington JR (1975) Mutual interference between parasites or predators and its effect on searching efficiency. J Anim Ecol 44:331-340

Cantrell RS, Cosner C (2003) Spatial ecology via reaction-diffusion equations. Wiley, Hoboken

Chen F, Chen Y, Shi J (2008) Stability of the boundary solution of a nonautonomous predator-prey system with the BeddingtonDeAngelis functional response. J Math Anal Appl 344:1057-1067

Cui J, Takeuchi Y (2006) Permanence, extinction and periodic solution of predator prey system with Beddington-DeAngelis functional response. J Math Anal Appl 317:464-474

DeAngelis DL, Goldstein RA, Neill VO (1975) A model for trophic interaction. Ecology 56:881-892

Dimitrov DT, Kojouharov HV (2005) Complete mathematical analysis of predator-prey models with linear prey growth and Beddington-DeAngelis functional response. App Math Comput 162:523-538 
Gao W, Baskonus HM, Shi L (2020a) New investigation of batshosts-reservoir-people coronavirus model and application to 2019-nCoV system. Adv Differ Equ 391:2020

Gao W, Veeresha P, Baskonus HM, Prakasha DG, Kumar P (2020b) A new study of unreported cases of 2019-nCOV epidemic outbreaks. Chaos Solitons Fractals 138:109929

Gao W, Veeresha P, Prakasha DG, Baskonus HM (2020c) Novel dynamic structures of 2019-nCoV with nonlocal operator via powerful computational technique. Biology 9:107

Gao W, Yel G, Baskonus HM, Cattani C (2020d) Complex solitons in the conformable $(2+1)$-dimensional Ablowitz-Kaup-NewellSegur equation. AIMS Math 5(1):507-521

Gauss GF (1935) Struggle of existence. Williams and Wilkins, Baltimore

Gazi NH (2012) Dynamics of a marine plankton system: diffusive instability and pattern formation. App Math Comput 218:8895-8905

Guin LN, Mondal B, Chakravarty S (2017) Stationary patterns induced by self- and cross-diffusion in a Beddington-DeAngelis predator-prey model. Int J Dyn Control 5:1051-1062

Holling CS (1965) The functional response of predators to prey density and its role in mimicry and population regulation. Mem Entomol Soc Can 45:5-60

Hwang TW (2003) Global analysis of the predator-prey system with Beddington-DeAngelis functional response. J Math Anal Appl 281:395-401

Legendre P, Fortin MJ (1989) Spatial pattern and ecological analysis. Plant Ecol 80:107-138

Levin SA, Segel LA (1976) Hypothesis for origin of planktonic patchiness. Nature 259:659-680

Li H, Takeuchi Y (2011) Dynamics of the density dependent predator-prey system with Beddington-DeAngelis functional responses. J Math Anal Appl 374:644-654

Liu QX, Li BL, Jin Z (2008) Resonance and frequency-locking phenomena in spatially extended phytoplankton-zooplankton system with additive noise and periodic forces. J Stat Mech 5:P05011

Lotka AJ (1924) Elements of physical biology. Williams and Wilkins, Baltimore

Luckinbill LL (1973) Coexistence in laboratory population of paramecium aurelia and its predator Didinium nasutam. Ecology 56:1320-1327

Maionchi DO, dos Reis SF, de Aguiar MAM (2006) Chaos and pattern in a spatial tritropic food chain. Ecol Model 191:291-303

Malchow H (1993) Spatio-temporal patterns formulation in nonlinear equilibrium plankton dynamics. Proc $\mathrm{R}$ Soc Lond $\mathrm{B}$ 251:103-109

May RM (1973) Stability and complexity in model ecosystems. Prinsceton University Press, New Jersey
Medvinsky AB, Petrovskii SV, Tikhonova IA, Malchow H, Li BL (2002) Spatio-temporal complexity of plankton and fish dynamics. SIAM Rev 44:311-370

Naji RK, Balasim AT (2007) Dynamical behavior of a three species food chain model with Beddington-DeAngelis functional response. Chaos Solitons Fractals 32:1853-1866

Parshad RD, Kumari N, Kasimov AR, Anderraahmane HA (2014) Turing patterns and long-time behavior in a three-species foodchain model. Math Biol 254:83-102

Parshad RD, Bhowmick S, Quansah E, Basheer A, Upadhyay RK (2016) Predator interference effects on biological control: the 'paradox' of the generalist predator revisited. Commun Nonlinear Sci Numer Simul 39:169-184

Pascual M (1993) Diffusion induced chaos in a spatial predator-prey system. Proc R Soc Lond B 251:1-7

Petrovskii SV, Malchow H (2014) Wave of chaos: new mechanism of pattern formation in spatiotemporal population dynamics. Theor Popul Biol 59:157-174

Sánchez YG, Sabir Z, Günerhan H, Baskonus HM (2020) Analytical and approximate solutions of a novel nervous stomach mathematical model. Discrete Dyn Nat Soc 5063271:9

Segel LA, Jackson JL (1972) Dissipative stucture: an explanation and an ecological example. J Theor Biol 37:545-559

Turing A (1852) The chemical basis of morphogenesis. Philos Trans

Upadhyay RK, Kumari N, Rai V (2009) Wave of chaos in a diffusive system: generating realistic patterns of patchiness in planktonfish dynamics. Chaos Solitons Fractals 40:262-276

Upadhyay RK, Naji RK, Raw SN, Dubey B (2013) The role of top predator interference on the dynamics of a food chain model. Commun Nonlinear Sci Num Simul 18:757-768

Upadhyay RK, Roy P, Venkataraman C, Madzvamuse A (2016) Wave of chaos in a spatial eco-epidemiological system: generating realistic patterns of patchiness in rabbit-lynx dynamics. Math Biol 281:98-119

Volterra V (1926) Fluctuations in the abundance of a species considered mathematically. Nature 118:558-560

Wonlyul K, Inkyung A (2013) A diffusive one-prey and twocompeting-predator system with a ratio-dependent functional response: I, long time behavior and stability of equilibria. J Math Anal Appl 397:9-28

Yel G, Baskonus HM, Gao W (2020) New dark-bright soliton in the shallow water wave model. AIMS Math 5(4):4027-4044

Zhang Y, Xu Z, Liu B, Chen L (2005) Dynamics analysis of a Holling I predator-prey system with mutual interference concerning pest control. J Biol Syst 13(1):45-58

Zhifu XI (2012) Cross-diffusion induced Turing instability for a three species food chain model. J Math Anal Appl 388:539-547 